\title{
THE EFFECT OF MEDITATION ON VISUAL AND AUDITORY SUSTAINED ATTENTION
}

\author{
by
}

Paige Badart

A thesis submitted to Victoria University of Wellington in fulfilment of the requirements for the degree of Master of Science in Cognitive and Behavioural Neuroscience

Victoria University of Wellington 2015 


\section{ACKNOWLEDGEMENTS}

I would like to thank Dr Steven Prime for his support and advice from across the globe during the production of this thesis. Another huge thank you to Dr John McDowall for his support and guidance. To all of who participated in the research, your enthusiasm and cooperation is truly appreciated. Finally, I would like to thank my partner, family and friends who have all been greatly supportive during the development of this thesis and throughout my years at university. 


\begin{abstract}
Failures of attention can be hazardous, especially within the workplace where sustaining attention has become an increasingly important skill. This has produced a necessity for the development of methods to improve attention. One such method is the practice of meditation. Previous research has shown that meditation can produce beneficial changes to attention and associated brain regions. In particular, sustained attention has shown to be significantly improved by meditation. While this effect has shown to occur in the visual modality, there is less research on the effects of meditation and auditory sustained attention. Furthermore, there is currently no research which examines meditation on crossmodal sustained attention. This is relevant not only because visual and auditory are perceived simultaneously in reality, but also as it may assist in the debate as to whether sustained attention is managed by modalityspecific systems or a single overarching supramodal system.
\end{abstract}

The current research was conducted to examine the effects of meditation on visual, auditory and audiovisual crossmodal sustained attention by using variants of the Sustained Attention to Response Task. In these tasks subjects were presented with either visual, auditory, or a combination of visual and auditory stimuli, and were required to respond to infrequent targets over an extended period of time. It was found that for all of the tasks, meditators significantly differed in accuracy compared to non-meditating control groups. The meditators made less errors without sacrificing response speed, with the exception of the Auditory-target crossmodal task. This demonstrates the benefit of meditation for improving sustained attention across sensory modalities and also lends support to the argument that sustained attention is governed by a supramodal system rather than modality-specific systems. 


\section{TABLE OF CONTENTS}

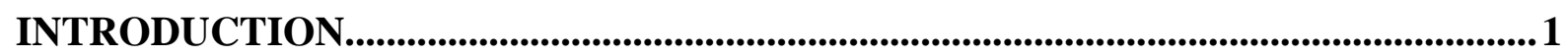

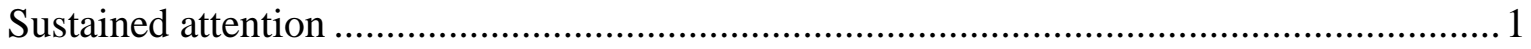

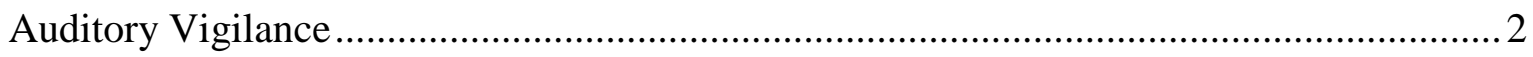

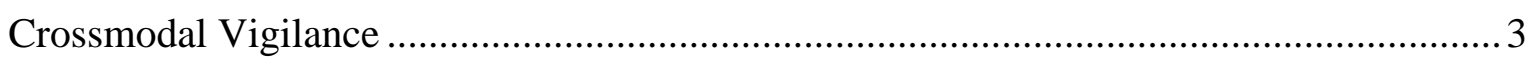

Sustained Attention to Response Task .......................................................................... 4

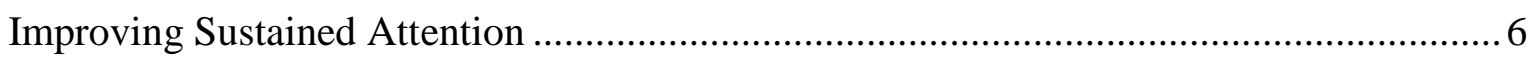

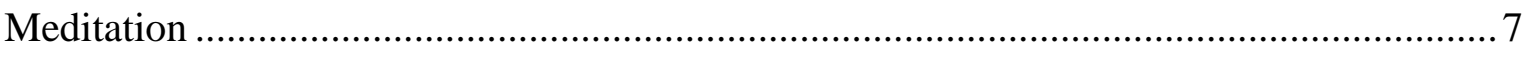

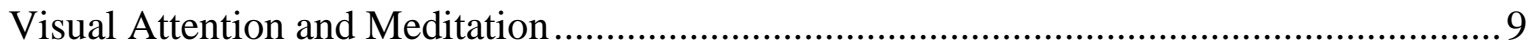

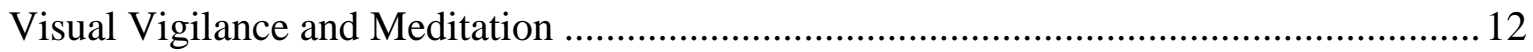

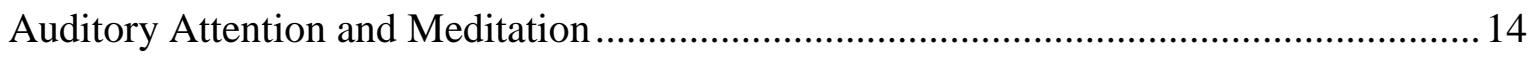

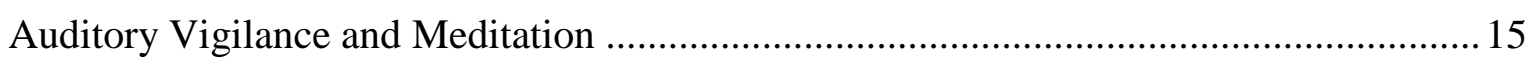

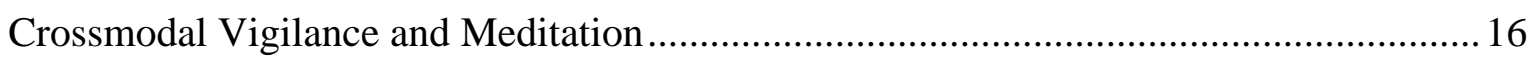

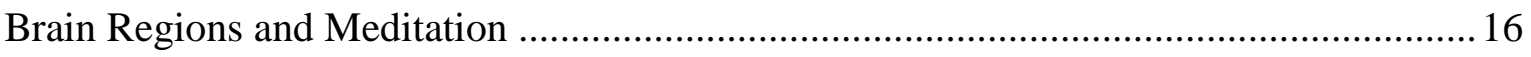

Differences between Focused Attention and Open Monitoring ....................................... 19

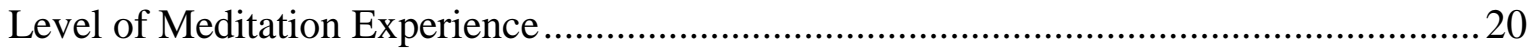

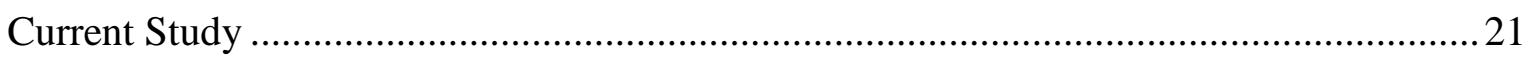

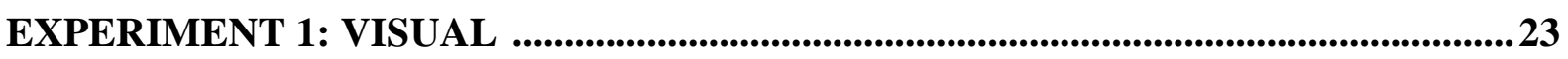

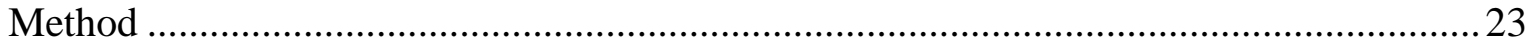

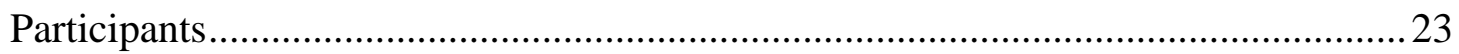

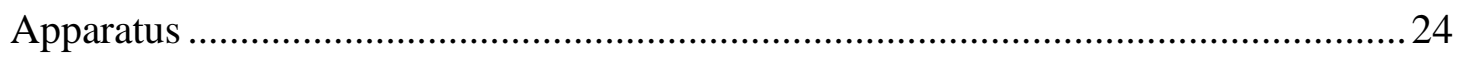

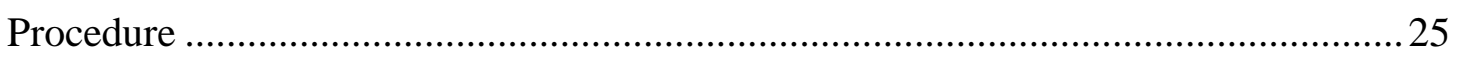

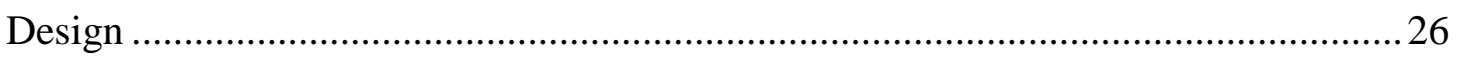

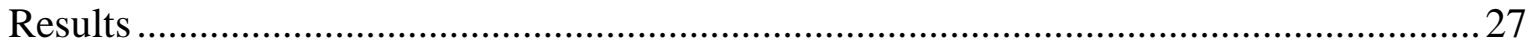

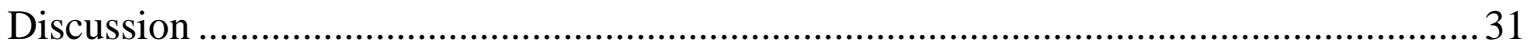

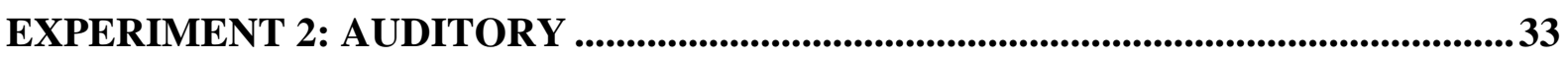

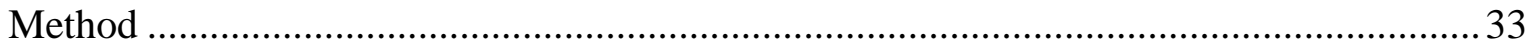

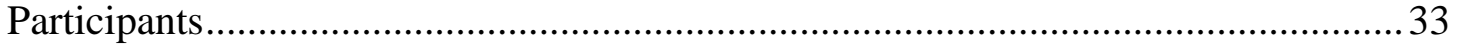

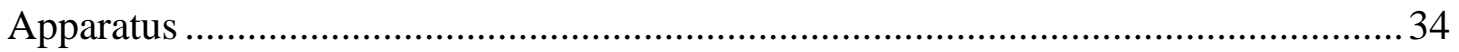

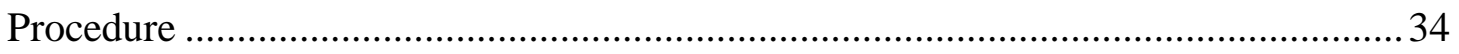




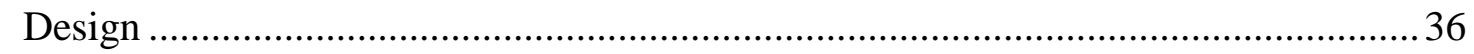

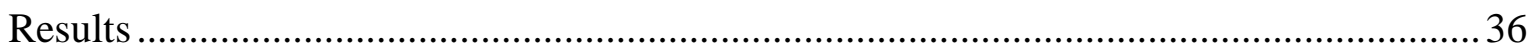

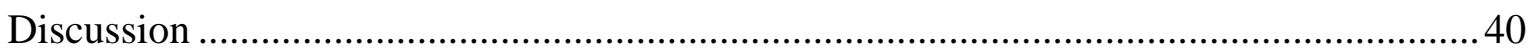

EXPERIMENT 3A: AUDITORY TARGET .....................................................................43

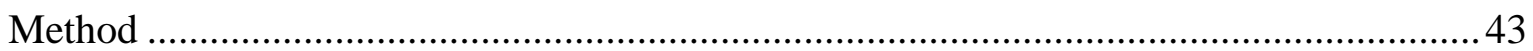

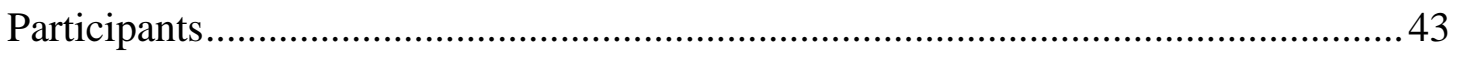

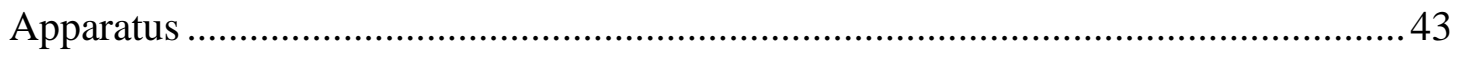

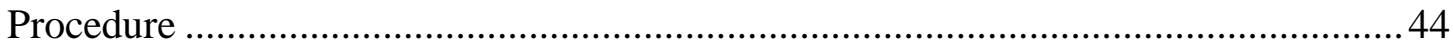

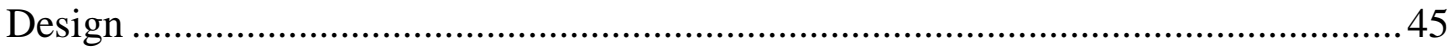

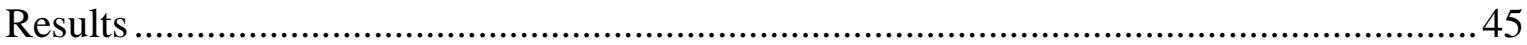

EXPERIMENT 3B: VISUAL TARGET ..........................................................................49

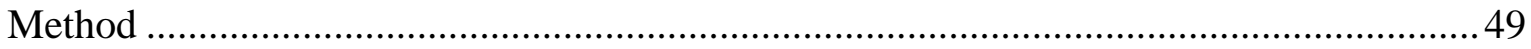

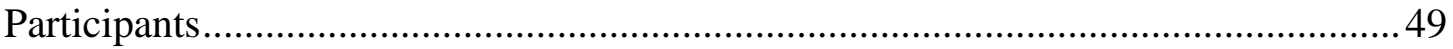

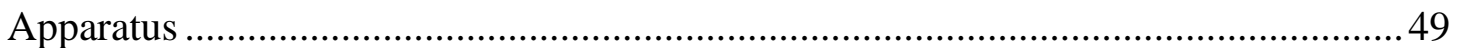

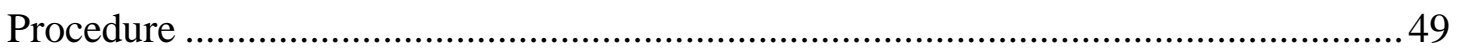

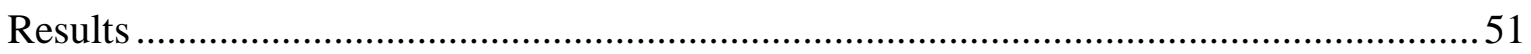

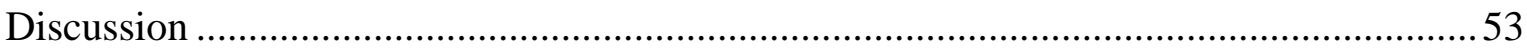

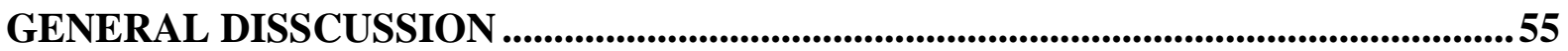

Modality-Specific versus Supramodal Model of Vigilance …………………………......57

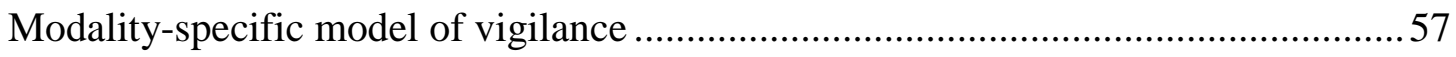

Supramodal model of vigilance ……………………….....................................5

Possible Explanations for Meditation-Induced Changes in Attention ...................................60

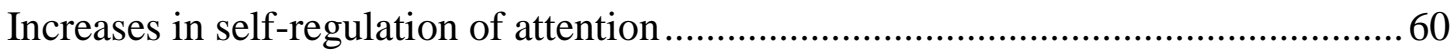

Improvements in executive control......................................................................... 61

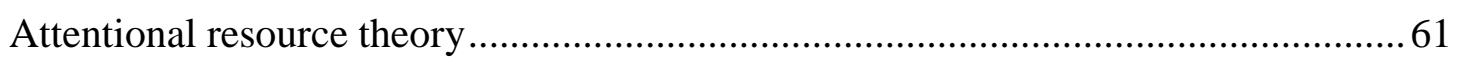

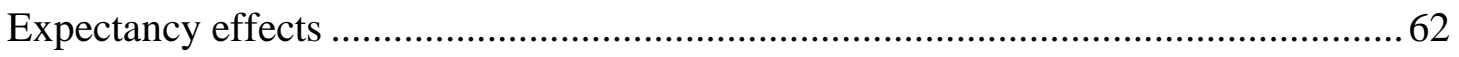

Self-related motivations ....................................................................................6

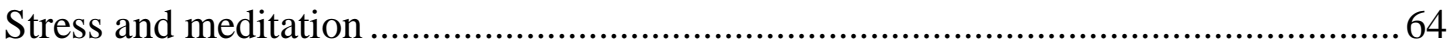

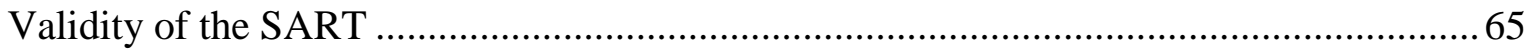

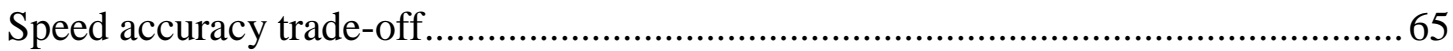

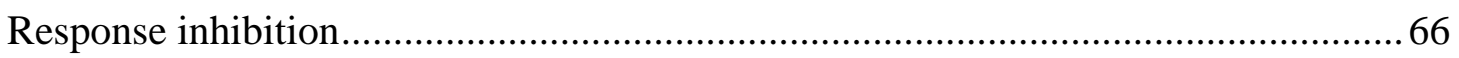


Implications

Concluding Remarks

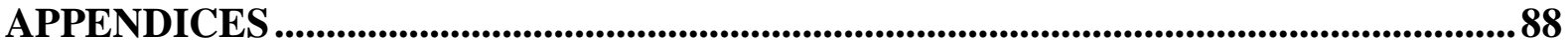

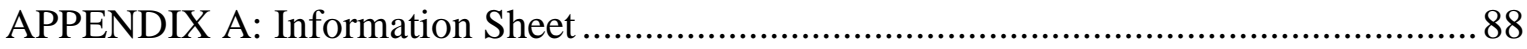

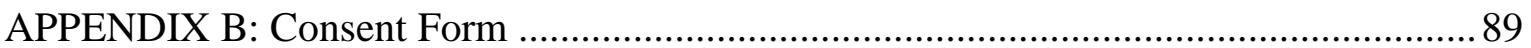

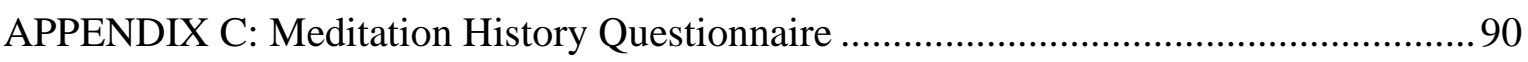

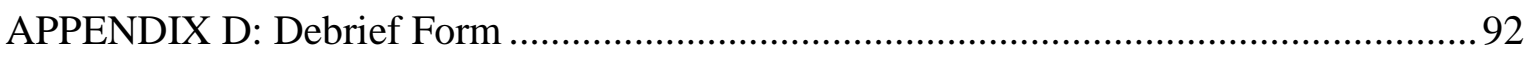

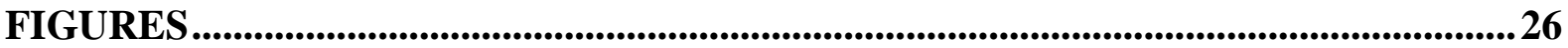

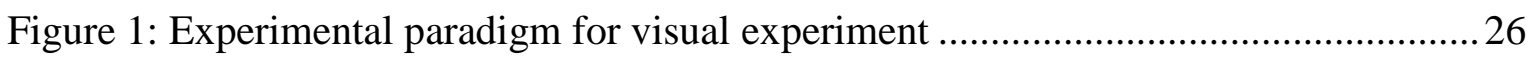

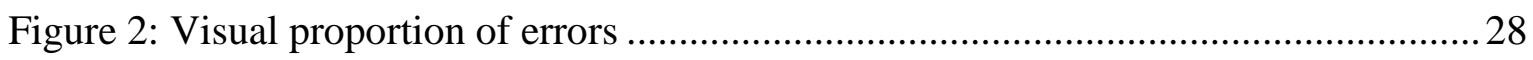

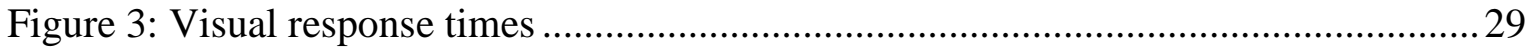

Figure 4: Experimental paradigm for auditory experiment …......................................... 35

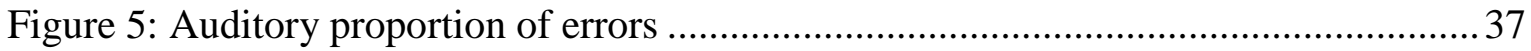

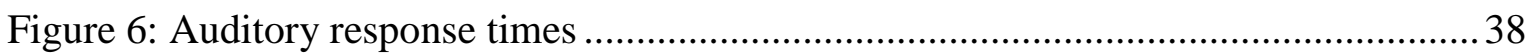

Figure 7: Experimental paradigm for auditory-target experiment ................................... 44

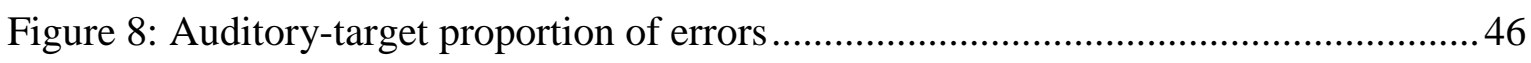

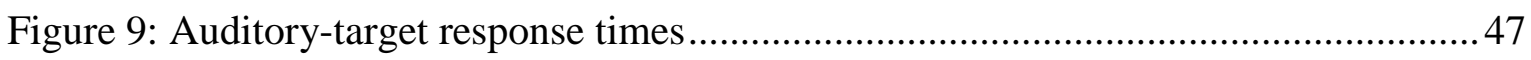

Figure 10: Experimental paradigm for visual-target experiment .......................................50

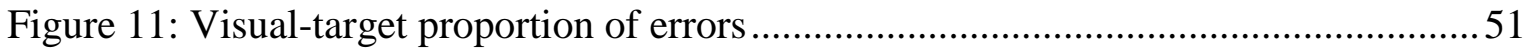

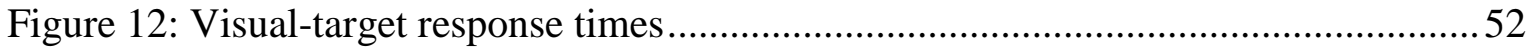


Lapses in attention are a common occurrence in everyday life. They frequently arise when attention has to be sustained for extended periods of time and alertness needs to be maintained (Warm, 1993). While some lapses in attention are merely inconvenient, others can be hazardous (Cheyne, Carriere \& Smilek, 2006). For example, driving long distances causes performance to decline with the duration of travel and failures in attention can cause injury or even be fatal (Barkley, 2007; Verster \& Roth, 2013). More traffic fatalities have been caused by attentional failures than by drugs, speed or fatigue (Knowles \& Tay, 2002).

Furthermore, sustaining attention has also become a highly important factor in many modern day careers. Advances in automated technology in the workforce have shifted the role of humans from active controllers to supervisors (Sheridan, 1980). The supervision of automated equipment is required in such roles as air traffic control, military surveillance, airport baggage inspection, and long-distance driving (Warm, Parasuraman \& Matthews, 2008). Failure to maintain attention in such fields can lead to losses in system productivity and can cause workplace injuries (Barger et al., 2006) This emphasises the serious implications of attentional failures and the need for research on sustained attention to determine methods to improve attention.

\section{Sustained Attention}

Sustained attention has been defined as the ability to self-maintain conscious processing of stimuli that is repetitive or non-arousing, and which would otherwise lead to habituation or distraction toward other stimuli (Robertson, Manly, Andrade, Baddeley \& Yiend, 1997). It is characterised by the ability to detect irregular relevant signals ('vigilance'). This ability declines over time and is known as the vigilance decrement (Sarter, Givens \& Bruno, 2001). Traditionally, the vigilance decrement was thought to occur due to a decline in arousal (Frankmann \& Adams, 1962). This theory assumed that the monotonous 
and under-stimulating nature of vigilance tasks caused suppression in brain systems associated with maintaining attention, leading to poorer performance. Yet this theory struggled to explain how numerous studies found poor performance across vigilance tasks that varied greatly in the stimuli presented, sensory modality and critical signals. It was unlikely that such a wide-range of tasks were under -stimulating. In light of this, it was suggested that the vigilance decrement may be due to the high demand on information processing resources (Warm et al., 2008).

Warm and colleagues (2008) proposed that the reductions in sustained attention might be better explained by attentional resource theory (Kahneman, 1973; Wickens, 1981). This theory explains the vigilance decrement as the reduction in availability of attentional resources that cannot be replenished in the available time period. A study showed that the vigilance decrement was not due to the tasks being under-stimulating, but rather due to the high workload of the tasks (Warm, Dember \& Hancock, 1996). This argument is also supported by research that showed a decrease in cerebral blood flow over time which parallels the performance decrement (Hitchcock et al., 2003). Therefore, the decline in vigilance over time can be explained by a concurrent decline in attentional resources.

\section{Auditory Vigilance}

While there is a large body of literature focused on visual vigilance, there is less focus on other sensory modalities such as auditory vigilance. There is a need for further research on this topic due to the high number of workplace operators requiring focused attention on infrequent auditory signals (Neal \& Pearson, 1966). Auditory vigilance is defined as the selfmaintenance of conscious processing of auditory stimuli that is either repetitive or nonarousing, and would usually lead to distraction or habituation (Robertson et al., 1997). 
Multiple studies have reported differences in task performance between visual and auditory vigilance. Overall, the vigilance decrement has shown to be reduced in auditory compared to visual tasks (Davies \& Parasuraman, 1982; Seli, Cheyne, Barton \& Smilek, 2012; Szalma et al., 2004), while the response times in auditory tasks have also shown to be slower (Seli et al., 2012). This could be due to differences in temporal processing. Auditory tasks have shown to have superior temporal accuracy (Repp \& Penel, 2002) and auditory intervals tend to be perceived as longer than visual intervals (Goldstone \& Goldfarb, 1966). Furthermore, there is little to no correlation in performance between visual and auditory vigilance tasks (Gruber, 1964; Loeb \& Binford, 1968).

In addition, stress has shown to impact more heavily on visual than auditory processing with participants reporting greater levels of stress during visual tasks (Galinsky, Rosa, Warm \& Dember, 1993) and showing greater recovery from stress during auditory tasks (Szalma et al., 2004). As higher stress levels are associated with poorer performance (Temple et al., 2000) this could further influence a difference in performance between visual and auditory vigilance.

Due to the differences between visual and auditory vigilance tasks, it could be proposed that there is a difference in the way vigilance is processed in the brain. It has also been suggested that because each modality has different transduction properties the ability to discriminate between signals needs to be equated across tasks in order to make them comparable (Curtindale, Laurie-Rose, Bennett-Murphy \& Hull, 2007).

\section{Crossmodal Vigilance}

Even fewer studies have investigated the effects of multiple modalities within the same vigilance task. While attention is generally studied in isolation in terms of sensory 
modality, this is far from reality where modalities are continuously linked to create a fluid perception of the world (Driver \& Spence, 1998).

With differences in performance between visual and auditory vigilance tasks, it could be assumed that they function through different attentional systems. Yet findings from other studies on intermodal vigilance performance suggest that vigilance might not be modality specific (Shaw et al., 2009; Warm \& Jerison, 1984). Tyler, Waag and Halcomb (1972) proposed that the low or non-significant correlations between visual and auditory vigilance may be due to differences in task difficulty. A study was conducted where they equated the task difficulty across visual-only, auditory-only and intermodal tasks. They found that all three tasks were highly correlated when signal discriminability was controlled for which suggests that vigilance may be a unitary construct across modalities.

\section{Sustained Attention to Response Task}

While there are many measures of vigilance that have been used, they rarely correlate with attentional slips in everyday life (Rabbitt \& Abson, 1990). Robertson and colleagues (1997) developed a new vigilance task, the Sustained Attention to Response Task (SART), with hopes of creating a measure with higher external validity. Typically, vigilance tasks require participants to monitor long sequences of stimuli and only make a response when an infrequent target is detected. Participants are then likely to enter a state of unfocused attention and rely only on reflexive detection mechanisms to perceive and respond to the infrequent target. These tasks also have problems with ceilings effects which leads researchers to increase working memory load in order to reduce the high levels of accuracy (Parasuraman, Mutter \& Molloy, 1991). In contrast, the SART requires participants to respond to a continuous stream of non-target stimuli, and withhold a response for an infrequent target. As participants are required to constantly respond, they develop a motor set which makes the 
inhibiting of a response more effortful. It is argued by Robertson and colleagues (1997) that this will require subjects to make controlled responses rather than automatic. This type of task is more sensitive to even minor lapses of attention, which dramatically increases the likelihood of making an error (Cheyne et al, 2006; Cheyne, Solman, Carriere \& Smilek, 2009).

The SART has shown to be a more sensitive and valid measure of sustained attention than traditional vigilance tasks. While the SART has been criticised for measuring response inhibition, performance on the SART can be predicted by performance on other measures of sustained attention. Robertson and colleagues (1997) also showed that performance on the SART was significantly correlated with self-reports of everyday attentional failures (Smilek, Carriere \& Cheyne, 2010). Finally, it also showed a significant difference between a group of traumatic brain injury patients and a matched control group, suggesting that the SART is sensitive to deficits in sustained attention.

Other studies have developed various forms of the SART. Cheyne and colleagues (2009) developed the response switching task which differs to the SART in that it required subjects to make a different key response when a target stimulus appeared, rather than inhibiting the response altogether. This variant produced similar error rates to the SART but also allows for further analysis of the trials where subjects failed to switch responding during the presentation of a target.

Another study by Seli and colleagues (2012) developed different versions of the SART to measure sensory modalities; auditory and multimodal. In the auditory SART they presented the digits one to nine spoken in rapid succession. The multimodal version consisted of the concurrent presentation of both visual digits and spoken digits, followed by both visual and auditory masks. They found that the auditory version produced significantly fewer errors 
than the visual task, while the multimodal had an intermediate number of errors. The response times were also much slower in the auditory version of the SART, which is consistent with traditional measures of auditory vigilance and suggests that subjects may have had more time when responding to recover from brief attention lapses. The multimodal version was similar in response times to the visual task. They also looked at RT variability, a measure sensitive to variations of response times over the course of the task, and found that there were no differences across all three tasks. As individual subjects performed similarly across all tasks and the correlations across tasks were as strong as within tasks, the three tasks were directly comparable and were measuring the same processes.

\section{Improving Sustained Attention}

As previously mentioned, the need to develop ways to improve sustained attention has become increasingly relevant. There are some methods available that have shown to do this. For example, a review by Koelega (1993) revealed that consumption of amphetamine, caffeine and nicotine improved overall performance on vigilance tasks and prevented the occurrence of a vigilance decrement; meaning that accuracy did not diminish over time. Furthermore, recovering the ability to sustain attention after injury is also possible. Sturm, Willmes, Orgass and Hartje (1997) showed that patients with unilateral vascular lesions could improve their accuracy on a vigilance task after a series of training sessions. This coincides with research on practice effects in vigilance tasks, which much like other attention tasks show improvements in performance with repeated execution (Parasuraman \& Giambra, 1991).

While these results seem positive, there are concerns over how these improvements in attention can be generalised to everyday activities (Kerns, Eso \& Thompson, 1999). Also, the consumption of stimulants can have other side effects that would outweigh any benefits to 
attention (Carr \& White, 1986). Furthermore, although training and practice on vigilance tasks improves detection of targets, the decline in performance over time still remains (Binford \& Loeb, 1966; Weiner, 1968). Research therefore needs to focus on alternative methods to improve sustained attention.

\section{Meditation}

A possible method for improving sustained attention is meditation. Meditation has been used for thousands of years within religious and philosophical contexts in the Eastern world, while only during the last few decades it has become an area of interest among psychology in the West (Shapiro \& Walsh, 1984). In recent studies it has shown promise in the treatment across many health domains including; physical and mental health, stress, drug consumption, depression, and eating disorders (Kristeller \& Hallett, 1999; Kuyken et al., 2008; Monk-Turner, 2003; Speca, Carlson, Goody \& Angen, 2000).

Since meditation is a broad term that includes a wide variety of techniques and practices, attempts have been made to create a more precise definition. Cardoso, Souza, Camano, and Leite (2007) have defined meditation as being a self-induced state that is applied through a specific technique, using self-focused skill, which aims for muscle and logic relaxation. In this definition, self-focused skill refers to the ability to maintain focused attention. This is achieved either by focusing on a single point or by leaving attention free by having no point of focus. The term logical relaxation refers to the ability to not judge, analyse or create expectations of any experiences during meditation practice. Therefore, the practitioner does not become caught up in any thoughts expressed during the meditation process. Attention has long been viewed as an important aspect of meditation (Di Nardo \& Raymond 1979; Van Nuys, 1971), however there is still a lack of research concerning the specific effects of meditation on attention. 
The term meditation refers to a wide variety of practices that range from types designed to allow relaxation, to other techniques that aim to increase well-being or even achieve enlightenment (Lutz, Slagter, Dunne \& Davidson, 2008). However, most practices are able to be categorised into two main branches. These categorisations are also commonly used in Western scientific research to operationalise meditation types. The first is focused attention (FA) meditation, also known as Samatha, which involves maintaining sustained attention upon a selected object. This object can be an image, mantra, or the sensation of breathe through the nostril (Lutz et al., 2008; Rizzi, 2005; Wallace, 2005). Subjects also engage in self-monitoring where attention must be gently directed away from distractors and intrusive thoughts and brought back to the object of focus. Progress can be determined by the level of effort required to maintain focus. While novices may find that distractions are frequent and constant effort is required, experienced practitioners require very little effort to maintain a high level of focus. In highly advanced practitioners this seems to become effortless (Lutz et al., 2008).

Interestingly, there are many similarities between ancient descriptions of the processes involved in FA meditation and modern cognitive explanations of attention (Lutz et al., 2008). Both identify that maintaining attention on an intended point of focus requires the ability to monitor attention by disengaging from distractions, and engaging attention toward the goal. The primary goal of meditation is to reduce the constant inner dialogue of the mind and to be fully aware in the present (Boorstein, 1996).

The other main branch of meditation is open monitoring (OM), also known as Vipassana or mindfulness meditation. Vipassana means to see with clarity and precision; to see true reality (Gunaratana, 1991). In contrast to FA, open monitoring does not involve selective attention on an object but rather an increased awareness of one's own thoughts and experiences with an attitude of non-judgemental curiosity and openness (Marchand, 2012). 
This type also has a component of attentional control, but subjects are encouraged to become an observer of their own thoughts, emotions and senses without placing judgement; accepting the present moment without trying to change anything (Lutz et al., 2008; Rizzi, 2005; Wallace, 2005). Many Western therapies are based on OM such as mindfulness-based stress reduction (MBSR) (Kabat-Zinn, 1982) and mindfulness-based cognitive therapy (MBCT) (Segal, Williams \& Teasdale, 2002).

These two branches of meditation are not always practiced independently. In fact, it is commonly expected that an individual must master the attentional training aspect as a prerequisite to training the non-judgemental attitude towards one's own experiences (Moore, Gruber, Derose \& Malinowski, 2012). It is also argued that there is a third category of meditation that does not fit into FA or OM. Transcendental meditation belongs to a distinct category that has been labelled automatic self-transcending (Josipovic, 2010). However, for the purposes of this study only FA and OM will be focused upon as the majority of previous research have used and compared these two types of meditative practice.

\section{Visual Attention and Meditation}

There is a body of literature that shows that individuals who meditate have superior attentional processing compared to non-meditators. Hodgins and Adair (2010) studied the effects of meditation on a range of attentional paradigms. Individuals from meditation centres were asked to complete a variety of perception and attention tasks, such as the change blindness flickering task (originally pioneered by Rensink, O’Regan \& Clark, 1997), the Gorilla video (from Simons \& Chabris, 1999), an ambiguous image perspective-switching task, and the classic attentional cueing task (Posner, 1980). They found that regular meditators; had a reduced change blindness (i.e. they detected a greater number of changes and detected these more quickly), had better visual concentration (counted moving stimuli 
more accurately in the gorilla video), had greater ability to shift focus and flexibly process images, and had better selective attention. This study suggests that meditation improves general attention across many domains.

Other studies have shown that specific types of attention may be more sensitive to improvements through meditation. Valentine (1988) found that meditators had significantly lower scores on the Everyday Attention Questionnaire (Martin, 1983) which suggests preferential use of focused attention over divided attention. Other studies supported this claim by showing that meditators performed better than controls on a dichotic listening task which requires focused attention to one ear while ignoring stimuli from the opposing ear (Martin, 1978).

On the other hand, some studies have shown that meditation effects executive control over other types of attention (Ainsworth, Eddershaw, Meron, Baldwin \& Garner, 2013; Tang et al., 2007). Tang and colleagues (2007) conducted a study where subjects participated in five days of integrative body-mind training (IBMT) and were examined using the Attention Network Test on alerting, orienting and executive control. Alerting refers to maintaining alertness, orienting refers to directing attention toward a target, and executive control refers to high-level processing such as conflict resolution between competing stimuli. The results showed that compared to a relaxation control group, the meditation group showed improvements in executive functioning but not alerting or orienting.

A further study by Moore, Gruber, Derose and Malinowski (2012) used a Stroop Word-Colour task (Stroop, 1935) to measure differences in executive control between meditators and non-meditators. The Stroop Word-Colour task involves naming a colour word that is printed in a conflicting colour (e.g. BLUE printed in red). To understand the neuronal processes that underlie this attentional benefit they used electroencephalograph (EEG) 
recordings and measured changes in the third positive deflection (P3) and second negative deflection (N2) of the event-related potentials. While there were no significant differences in behavioural performance between meditators and non-meditators, there was a significant decrease in P3 and increase in N2 for meditators compared to non-meditators. This was argued to indicate that meditators showed an increase in attentional processing of the word and a reduction of attentional resources required to process the stimuli (Moore, Gruber, Derose \& Malinowski, 2012).

Other research focused on the effects of meditation on a psychological phenomenon known as the attentional blink $(\mathrm{AB})$. This occurs when subjects are faced with a rapid succession of visual information at high temporal speeds and are required to detect two target stimuli among a stream of distractors. Subjects often fail to detect the second target when it is presented within 200-500ms after the first target (Chun \& Potter, 1995; Wyble, Bowman \& Nieuwenstein, 2009). In a study by Van Vugt and Slagter (2014), participants were required to engage in four minutes of meditation prior to the $\mathrm{AB}$ task. Results indicated that the attentional blink was reduced after OM compared to FA meditation. However, this effect was only found in highly experienced meditators. It has been suggested that the attentional blink phenomenon is due to excessive amounts of attentional resource being allocated to the first target leaving inadequate resources for the second target to be detected (Chun \& Potter, 1995; Wyble, Bowman \& Nieuwenstein, 2009). This is also supported by EEG research (DelgadoPastor, Perakakis, Subramanya, Telles \& Vila, 2013; Slagter et al., 2007). The fact that meditators showed significantly less of an $A B$ may suggest that they are better able to spread resources among the stimuli. This gives further support to the argument that meditators are better able to manage attentional resources.

While these studies show that meditation has a positive influence on attention, there is a lack of consensus on what type of attention receives the greatest benefit. Some studies 
found that focused attention is more sensitive to changes; yet others suggested that executive control may be affected more by meditation. However, this was not consistent across all studies. Further research is required to determine the extent to which particular attentional systems are improved through meditation. In addition, this research suggested that meditators may have an improved ability to manage attentional resources. As the vigilance decrement is suggested to be caused by a decline in attentional resources, it could be argued that meditators would perform better at vigilance tasks.

\section{Visual Vigilance and Meditation}

A main focus of meditation, particularly FA, is the maintenance of attention on a target while ignoring distractors over long periods of time. Expert meditators generally have over 10,000 hours practice and are said to meditate for extended periods of time (Lutz et al., 2008). It could then be expected that people with advanced meditative skills would be able to sustain high levels of focused attention for long periods of time in comparison to those who have little or no meditation experience.

A study of self-reported mindfulness showed that higher levels of mindfulness were negatively related to target omissions on a test of visual vigilance (Schmertz, Anderson \& Robins, 2009). This means that higher self-reported levels of mindfulness in a normal population (i.e. those who do not regularly practice meditation) are associated with superior sustained attention.

A group of studies have also looked at populations who regularly meditate and examined their ability to sustain attention. For example, Chambers, Lo and Allen (2008) examined sustained attention in meditators using an Internal Switching task. In this task participants were shown words from one of two categories (food and household objects) and were told to keep a mental count of how many words they had seen from each category. As 
soon as they had seen the word and mentally counted it they were required to make a response. Response times were then analysed. It was found that meditators had significantly faster response times than controls.

MacLean and colleagues (2010) conducted a longitudinal study that tested the sustained attention of meditators. Participants attended a three month meditation retreat and practiced FA for five hours a day. A sustained visual attention task was used before, during and after training. In this task participants were presented with either a long or short line and were required to respond when they perceived the short line. The length of the short line was set at a personal threshold where the subject was able to detect it at $75 \%$ accuracy. They found that compared to a control group, meditators showed enhanced visual discrimination (i.e. a lower threshold between the short and long line) that coincided with previous research on improvements in visual perception (Brown, Forte and Dysart, 1984). They also found significant reductions in the vigilance decrement for meditators compared to the control group. MacLean and colleagues (2010) suggested that meditation training reduced the amount of resources required to discriminate between targets which then led to an increased level of resources available for sustaining voluntary attention.

These behavioural studies suggest that meditation improves the ability to sustain visual attention. While these are promising results, there has been very little consistency in the methodologies used across the research. The studies differed in the vigilance task, the type of meditation, frequency and duration of meditation practice, and whether research focused on state or trait. Studies that have looked at state examine meditators during or immediately following a short period of meditation and are interested in the behavioural or neurological changes that occur during this period. The other type of research involves studying the long term behavioural and neurological changes or traits of meditators. Although state research has benefits such as providing insight into brain functioning during meditation, 
the current research will focus on traits in order to determine the long term effects of meditation on sustained attention.

\section{Auditory Attention and Meditation}

While many studies have examined the effects of mediation on visual attention, there are very few that have focused on meditation and auditory attentional processing. Most of these studies focused on neural measures of attention such as EEG recordings. An early study focused on alpha recordings of long-term Zen meditators during the presentation of repetitive but infrequent auditory clicks (Kasamatsu \& Hirai, 1966). Alpha waves are usually present during relaxed wakefulness with closed eyes. When eyes are opened this leads to a decrease in alpha known as alpha blocking, which has been associated with active processing of stimuli (Niedermeyer, 1997). They found that while control participants showed an expected decrease in alpha blocking with the successive presentation of auditory clicks (indicating a habituation to the stimuli), experienced Zen practitioners had no decrease in alpha after each click. It was concluded that meditators were not habituating to the clicks and rather perceived each stimulus as novel, while not becoming distracted by them.

A recent study by Cahn, Delorme and Polich (2013) used an auditory oddball task with EEG recordings to determine whether meditators would show a decrease in automatic attentional engagement compared to controls. The auditory oddball task included frequent standard tones, infrequent oddball tones and infrequent distractor white noise bursts. Participants were asked to ignore the tones as best as possible and continue an OM style meditation during the task. The results showed that during meditation there was a lack of habituation to the frequent standard tones. This was indexed by an increase in early alpha power and theta phase-locking compared to controls during presentation of the standard tones. Early alpha power has been shown to be related to attentional engagement (Hanslmayr 
et al., 2007; Klimesch et al., 2004) and theta phase-locking has shown to be related to attentional engagement and incorporation of sensory stimuli into consciousness (Kahana, Seelig \& Madsen, 2001). This supports the argument that meditation practitioners do not habituate to repetitive stimuli. Therefore, they may be less susceptible to lapses of attention or mind wandering.

\section{Auditory Vigilance and Meditation}

Very few studies have focused on meditation and auditory sustained attention. Lutz and colleagues (2009) aimed to look at the longitudinal effects of meditation on sustained attention in both visual and auditory modalities. To measure this they used the attentional blink task to examine visual attentional processing and a dichotic listening task to examine auditory attentional processing. In the dichotic listening task subjects were asked to attend to tones and respond when they detected an occasional deviant tone among the frequent standard tones. They found that after a three month meditation retreat, more experienced meditators showed a significant decrease in the variability of their response times compared to a novice group. They also found an increase in phase consistency of theta-band oscillatory neural responses over anterior scalp regions, which also predicted the decrease in response time variability. Theta has been linked to cognitive control and the forming of mental representations important for sustaining attention (Hanslmayr et al., 2007; Jensen \& Lisman, 1996). These findings suggest that meditation increases response time precision on an auditory sustained attention task and increases neural processing associated with sustained attention.

Valentine and Sweet (1999) also conducted a study using an auditory vigilance task to examine differences in meditation styles on sustained attention. They employed the Wilkins counting test (Wilkins, Shallice \& McCarthy, 1987) which is a measure of sustained attention 
using auditory bleeps. It contains sets with bleeps ranging in number from 2 to 12 presented at different speeds. Participants are required to count and record the number of bleeps per set. Errors are more common during slower rates of presentation. They found that meditators made fewer errors than the control group. This suggests that meditators may have benefits for both visual and auditory sustained attention.

While there is a lack of research on meditation and auditory attentional processing, particularly sustained attention, the previous behavioural and neural evidence support the argument that meditators have greater advantage for auditory vigilance performance than non-meditators.

\section{Crossmodal Vigilance and Meditation}

To date there have been no studies of the effects of meditation on cross-modal vigilance. Visual and auditory vigilance have only been examined in isolation in regards to meditation. Research in this field would determine whether meditation improves vigilance for each sensory modality independently or if it generally improves vigilance over both sensory

modalities. It would also assist in the debate about whether attention systems are a unitary construct that govern all sensory modalities, or if there are modality-specific systems of attention.

\section{Brain Regions and Meditation}

While behavioural studies have shown that meditation influences the ability to sustain attention, there are also a number of brain regions that have shown to change in structure and efficiency after periods of meditation practice. Unsurprisingly, these regions have also shown to be associated with vigilance performance, independent of other cognitive and sensory 
processing. These changes provide supporting evidence for the argument that meditation improves the ability to sustain attention across modalities.

One area involved in sustained attention is the corpus callosum. Studies have shown that patients with a separated corpus callosum have trouble maintaining attention over extended periods (Rueckert, Sorenson \& Levy, 1994). Studies have also found correlations between callosa size and performance on vigilance tasks (Rao, Leo, Haughton, AubinFaubert \& Bernardin 1989). Furthermore, a study by Rueckert, Sorenson and Levy (1994) showed that collosa efficiency was significantly associated with faster performance on a vigilance task in a population of children. Therefore, the corpus callosum is a brain area is strongly associated with sustained attention.

Several studies have shown that the corpus callosum increases in connectivity after meditation practice. A study showed that only 11 hours of Integrative Body-Mind training spread over a month was enough to significantly increase the connectivity of the corpus callosum among novice practitioners (Tang et al., 2007). Luders and colleagues (2012) found that the connectivity of the corpus callosum (specifically the forceps minor) was significantly larger in a group of meditators than a group of age matched controls. There was no significant difference in the forceps major which suggests that meditation more heavily influences anterior regions of the brain.

There is also evidence to suggest that frontal regions are associated with sustained attention. Wilkins, Shallice and McCarthy (1986) found that patients with anterior lesions had poorer performance on a vigilance counting task than patients with posterior lesions. The right prefrontal cortex has shown to be associated with performance on sustained attention tasks across multiple modalities. Positron emission tomography (PET) studies have shown that the right prefrontal cortex is activated during both visual and auditory vigilance tasks 
(Cohen, Semple, Gross, King \& Nordahl, 1992; Pardo, Fox \& Raichle, 1991). These studies support the notion that vigilance is transferrable across modalities instead of being a separate process for each sensory modality.

Meditation has shown to change the structure of frontal regions in the brain. A study looking at grey matter concentrations found that practitioners of Vipassana had an increase in the right middle and superior frontal cortex (Hölzel et al., 2008). This study also found a correlation between grey matter concentrations in the orbitofrontal cortex and the amount of cumulative hours spent meditating. Furthermore, Lazar and colleagues (2005) found that regular meditation also significantly slowed age-related thinning of the prefrontal cortex. This suggests that meditation brings about beneficial changes to the frontal regions of the brain which may in turn lead to improvements in cognitive functioning and sustained attention across multiple modalities.

Another part of the brain associated with sustained attention is white matter connections. A study by Semrud-Clikeman and colleagues (2000) found that in children with ADHD, poorer performance on vigilance tasks was associated with smaller volume of right hemispheric white matter. Furthermore, a study showed that performance on a visual vigilance task was positively associated with connectivity of white matter in the right cingulum (Takahashi et al., 2010).

Tang and colleagues (2010) conducted a study that looked at the changes of white matter in individuals completing integrative body-mind training (IBMT). They found that subjects were able to significantly increase the connectivity of white matter compared to a control group who practiced non-meditative relaxation. However, these changes have shown to take time. Tang and colleagues (2010) found changes in connectivity of white matter only in individuals who practiced IBMT for 11 hours a day (for over a month), but did not find significant results for individuals who practiced for three or six hours per day. 
In sum, the regions of the brain predominantly argued to be involved in sustaining attention have all shown to increase in size and functioning for experienced (and relatively novice) meditators. This provides strong supporting evidence for the idea that meditation practice increases the ability to sustain attention.

\section{Differences between Focused Attention and Open Monitoring}

It has also been suggested that there are differences in the way attention is improved depending on the type of meditation practiced. Several studies have examined the differences in attentional processing between FA and OM meditation. A study by Jha, Krompinger and Baime (2007) examined performance on the Attentional Network Test (ANT) between; experienced FA practitioners (who then attended a three month mindfulness retreat), novice practitioners of mindfulness-based stress reduction (MBSR), and a non-meditating control group. Each group performed the ANT twice. The first test revealed that the FA practitioners had better executive control than the other groups. In the second test the FA practitioners who had attended the mindfulness retreat showed increases in their alerting performance, which was correlated with meditation experience. The MBSR group improved their orienting performance relative to the control group which showed no improvements. These results suggest that FA and OM may benefit different aspects of attention.

Not all studies have found a significant difference between these two distinct branches of meditation. Ainsworth and colleagues (2013) also compared FA and OM meditation in alerting, orienting, and executive control. They hypothesised that both FA and OM would increase executive control but not alerting or orienting. They also predicted that FA would have a greater benefit due to its sole focus on attention compared to OM, which jointly focuses on attention and emotional control. Results showed that both OM and FA improved their executive functioning compared to a control group; yet there was no difference between 
OM and FA as expected. This was proposed to be due to the overlap between both styles in developing skills, particularly for novice practitioners as used in this study.

In a recent study, Van Vugt and Slagter (2014) compared the magnitude of the attentional blink between FA and OM practitioners. The results showed that there was a smaller attentional blink for OM compared to FA meditators. This may be due to the ability of OM practitioners to better spread attentional resources, as a core focus of the practice is to maintain fluid awareness of stimuli. However, the association between OM and the attentional blink was only true for highly experienced meditators.

These studies show that while FA and OM may have different influences on attention, there is very little consistency across studies. However, the effect of experience level may assist in explaining some of the discrepancies between previous studies. Furthermore, there is a high level of overlap in early stages of practice between these two styles (Kapleau, 2013), which may have masked any significant differences.

\section{Level of Meditation Experience}

Not only does the type of meditation have an effect on the benefits to attentional processing, but also the length of time spent meditating. As mentioned, van Vugt and Slagter (2014) found a reduced attentional blink for meditators but this effect was only found for those with a high level of experience (on average 10,704 hours), suggesting that practice may be a crucial component to finding differences in attentional benefits.

Another study by Brefczynski-Lewis, Lutz, Schaefer, Levinson and Davidson (2007) used magnetic resonance imaging to examine brain activations in novice, experienced and expert meditators during sessions of meditation and rest. It was found that while all meditators showed brain activation in regions that are associated with attention and visual 
processing, activation differed in terms of strength and time course across subjects. While experienced meditators showed greater brain activation than novices; experienced meditators with fewer hours of practice had greater and more rapid activation than expert meditators with more hours of experience. This activation pattern fits an inverted-u shape and coincides with texts that describe concentrative meditation as being strenuous at first and then becoming effortless with practice (Lutz, Slagter, Dunne \& Davidson, 2008).

While these findings would suggest that only experienced meditators can reap the attentional benefits of meditation, studies have shown meditation training can improve attention in novice subjects. Tang and colleagues (2010) showed that 11 hours of Integrative body-mind training (IBMT) could significantly increase the connectivity of white matter tracts, and increase executive functioning in the Attention Network Test (Tang et al., 2007). Yet another previous study (Tang, 2009) found that only three hours of IBMT was enough to reduce the time required to resolve conflict in the Attention Network Test and increase activation in the anterior cingulate cortex. These studies suggest that some benefits to attentional processing can be acquired rapidly with comparatively little training.

\section{Current Study}

It is clear that meditation has some influence on cognitive and attentional processing. While there is much research dedicated to visual sustained attention there is a gap in the literature on both auditory and crossmodal sustained attention. As individuals do not use each sensory modality independently but rather blend them to perceive a unified reality, the lack of research on certain modalities can be detrimental to understanding how vigilance processes work within the brain. Furthermore, there is an obvious need for practical ways to improve sustained attention across all modalities for safety and productivity within the workplace. Both behavioural and neuropsychological evidence supports the argument that meditation 
practice improves performance on vigilance tasks and increases efficiency in associated brain regions. However, there is little cohesion across studies in the type of vigilance tasks used and the type and frequency of meditation practiced which has led to discrepancies in findings. Further research should focus on unifying these factors and determining the impact of meditation on vigilance across different sensory modalities.

The current study aims to research the effects of meditation, both focused attention and open monitoring, on vigilance performance as measured by the Sustained Attention to Response Task. This will be examined in visual and auditory modalities, as well as crossmodally with the concurrent presentation of visual and auditory stimuli. The following hypotheses are based on the previous research and aim to fill a gap in the literature surrounding meditation and vigilance across modalities.

From the analysis of previous studies on meditation and sustained attention it is expected that a group of meditators will have superior performance on a task of sustained attention compared to a control group. It is expected that they will be more accurate with a decrease in vigilance decrement, meaning that they will make fewer errors in the SART than their non-meditating counterparts. This is expected not only for the visual SART, but also for the auditory and cross-modal versions of the SART. It is also expected that these differences will not be due to strategic responding and therefore the response times will not significantly differ between meditators and controls. With regards to previous research, it is predicted that there may be a difference in performance between individuals associated with different types of meditation, with OM meditators making fewer errors than FA meditators due to the increase in their ability to spread attentional resources. However, this association may be influenced by the level of meditation experience. Finally, it is also expected that meditators with greater experience (as measured by time spent meditating) will make fewer errors than novice meditators with less experience. 
The following experiments aim to investigate these hypotheses. In Experiment 1 meditators will be compared to non-meditators in a visual-only version of the SART. Here, both target and non-target stimuli will be presented visually. Experiment 2 will follow the same general experimental paradigm, but instead of visual stimuli, subjects will be presented with auditory targets and non-targets. Finally, two crossmodal experiments will be included which both follow the same general experimental paradigm. In Experiment 3A subjects will be presented with auditory target stimuli and visual non-target stimuli. Conversely, Experiment 3B will include visual target stimuli and auditory non-target stimuli.

\section{Experiment 1: Visual}

\section{Method}

Participants. Two groups of participants were included in this study; a meditation group and a control group of non-meditators. The meditation participants were recruited through flyers posted at meditation classes from the Wellington region. Contact was initially made through email and then interested parties were visited and given a verbal explanation of what their participation in the study would entail. Each participant received a movie voucher for their time. Participants self-reported normal or corrected vision and hearing. A total of 22 (11 males and 11 females with a mean age of 37.1 and age range of 20-65 years) meditators participated in the experiment.

A control group of non-meditators was recruited primarily from Victoria University of Wellington. Before participating they were screened for no previous meditation experience and self-reported normal or corrected vision and hearing. Psychology students were offered course credit for their participation. All other control participants were offered a movie voucher for their time. The control group of non-meditators consisted of 22 participants (8 males and 14 females with a mean age of 37.64 and age range of 22-79 years). All 
participants received detailed information regarding the study (APPENDIX A) and gave written consent (APPENDIX B) before beginning the experiment.

Apparatus. The task was completed on a Dell Precision T1650, 3.30GHz using the programming software Psychopy (Peirce, 2007). The stimuli were presented on a 23 inch $120 \mathrm{~Hz}$ Samsung LCD monitor in a private room. A chin rest was mounted on the table and was $60 \mathrm{~cm}$ away from the screen.

Stimuli. The visual task was developed from the method used in a study by Cheyne, Ferrari and Cheyne (2012). It involved the rapid presentation of numbers ' 1 ' - ' 9 ' on the screen. An infrequent target stimulus ('3’) was pseudo-randomly presented at various times throughout the task. There was an equal probability of 1,3,5 or 7 non-target stimuli between each target. A target stimulus was never presented immediately after another target. The succession of trials was rapid with each stimulus only presented for $250 \mathrm{~ms}$, followed by a visual mask ('\&' symbol) displayed for $900 \mathrm{~ms}$ to reduce any after-images (Breitmeyer \& Öğmen, 2006). Thus each trial only lasted 1.15 seconds. A total of 1000 trials were included, 200 of which were target trials, giving a proportion of $20 \%$ target trials. Each stimulus was presented in Times New Roman font and in one of four randomised font sizes. This was to facilitate processing of the actual numerical digit and to reduce the possibility of participants using a perceptual template to identify the target stimulus (Robertson et al., 1997).

Meditation history questionnaire. A short questionnaire was given to meditation participants to determine their meditation history (APPENDIX C). This included questions developed from Grant, Courtemanche, Duerden, Duncan and Rainville (2010); the type of meditation practiced, number of years practicing, frequency of sessions per week, duration (in minutes) per week, time spent (in minutes) per session, time spent at retreats and motivation for practicing meditation. It also included questions designed to determine if 
participants were more closely aligned to concentrative (FA) meditation or mindfulness (OM) meditation (Valentine \& Sweet, 1999). The FA questions included "I focus my attention as far as possible to a single point- a mental image, a perceptual object, breath, sound or thought" and "I try to concentrate solely on this one item to the exclusion of everything else" while the OM questions included "I expand my attention/awareness to as many events as possible" and "I consider nothing a distraction". These were measured on a 5-point Likert scale, with 1 representing Strongly Disagree and 5 representing Strongly Agree.

Procedure. Upon arrival the participants were given an information sheet and offered as much time as necessary to read it and ask questions about their involvement in the study. All participants then signed a consent form. Meditation participants were given the questionnaire regarding their meditation history. The participants were then asked to sit comfortably in front of the computer with their head sitting in the chin rest. This was designed to keep participants a constant distance from the screen and to ensure that attention was maintained. They were asked to read the instructions on the computer screen. These were reinforced with verbal instructions and opportunity was given for questions to be asked. The task began with a set of ten practice trials. When participants felt comfortable with the task they could begin the experiment. Participants were instructed that speed was just as important as accuracy in this task, in that they should respond as quickly as they can and also as accurately as they can.

Participants performed a version of the SART task (Robertson et al., 1997). Figure 1 shows the general experimental paradigm. In a non-target trial, subjects were presented with a randomly assigned number (1-9, with the exception of 3) for 250ms. A mask ('\&' symbol) then appeared for $900 \mathrm{~ms}$. In a target trial, subjects were presented with the number ' 3 ' for $250 \mathrm{~ms}$, followed by a mask (' $\&$ ') for $900 \mathrm{~ms}$. In both trials, participants were required to make a response before the end of the trial (lasting $1150 \mathrm{~ms}$ ). This task lasted approximately 22 minutes. 


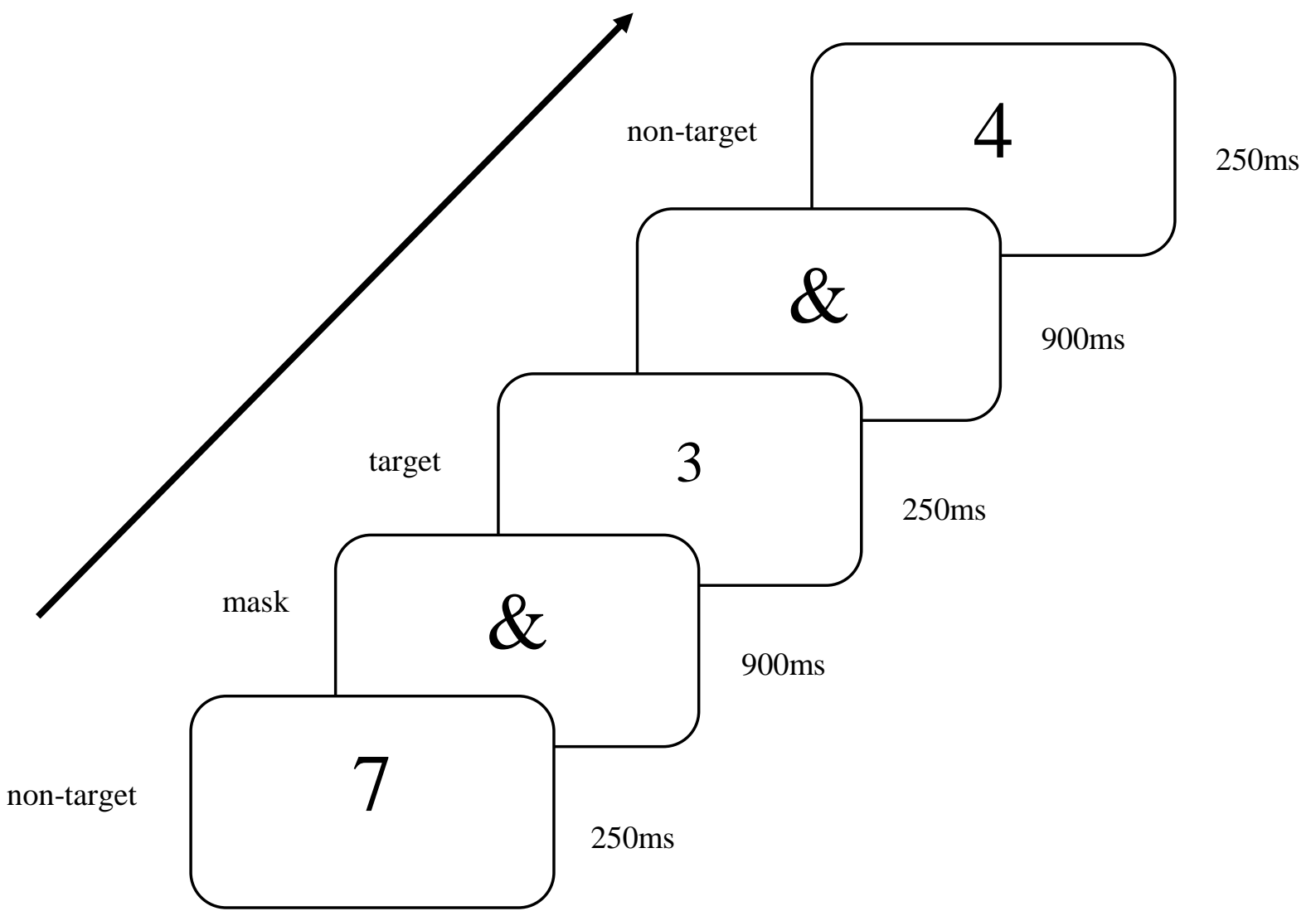

Figure 1. Experimental paradigm for visual experiment.

Responses. Participants responded to targets and non-targets by pressing either the left or right arrow key. Half of the participants were instructed to press the left arrow key for non-targets and the right arrow key for targets and vice versa for the other half of participants. The assignment of the keys to stimuli was counterbalanced between participants. The study by Cheyne and colleagues (2012) found that there was no significant difference in performance between using one hand or multiple hands in the SART. Therefore participants were able to press in a manner they found most comfortable (however the majority used the prescribed manner above). Handedness was recorded.

Design. This study used a between-subject design with meditation experience (meditators vs. non-meditators) as the independent variable. The primary dependent variable of interest was the proportion of errors made. Four measures of reaction time (RT) were included which represented the four possible responses (as per Cheyne et al., 2012): Default 
$R T$ was the RT for correct responses to a non-target stimulus, Switch $R T$ for target stimulus, Error $R T$ when pressing the non-target key when target was presented, and False alarm $R T$ when pressing the target key when non-target was presented. The reporting of reaction times alongside error rates are important in the SART as it has been shown to be susceptible to speed-accuracy trade-offs (Seli et al, 2012) where improvements in accuracy may be due to delayed responding. Therefore both accuracy and RT will be compared between groups.

\section{Results}

Firstly, analyses were conducted to ensure that the meditation and control groups did not differ in terms of age, gender or level of education. The meditation group had a mean age of $37.09(S D=12.34)$ and the control group had a mean age of $37.64,(S D=14.61)$. An Independent T-test showed that the two groups did not differ significantly in terms of age, $t$ $(42)=.134, p=0.89$. The meditation group had an equal number of males and females (11 males and 11 females) while the control group had slightly more females (8 males and 14 females). A chi-squared showed that these two groups did not significantly differ by gender, $\chi^{2}(1, N=44)=1.504, p=0.22$. Finally, education was examined by scoring participants selfreported highest level of education. High school qualification was scored as 1, trade certificates or equivalent was scored as 2, undergraduate degree was scored as 3 and postgraduate degree scored as 4 . A chi-squared showed that these two groups did not significantly differ by education, $\chi^{2}(3, N=44)=3.455, p=0.327$.

The purpose of this experiment was to determine if there are differences between meditators and non-meditators in a visual version of the SART. The SART tests vigilance, and thus, our primary interest is in their response accuracy, in particular if they were accurate in detecting a target when it was presented. The mean frequency of errors, failing to switch responses when the target was presented, in the meditation group were $33.59(S D=22.36)$ 
and in the control group $55.73(S D=31.63)$. False alarms were also included as an error where a non-target was presented on the screen and subjects incorrectly made a switch in responses. The mean frequency of false alarm errors were very low overall; $5(S D=7.03)$ and $4.23(S D=7.28)$ for meditators and non-meditators, respectively. Therefore, as with previous SART studies (Cheyne et al., 2012), false alarms will not be included in any further analyses.

It was hypothesised based on previous studies of visual vigilance and meditation that the proportion of target errors made would be significantly lower for meditators than nonmeditators. Overall, the mean proportion of errors made (an incorrect response to a target ' 3 ' trial) was greater for the control group, $27.46 \%(S D=16.19)$, than the meditation group, $16.82 \%(S D=12.20)$. This is shown in Figure 2. The proportion of errors made by the control group is comparable to results from previous studies that used the SART; where $\sim 30 \%$ errors were made on average (Cheyne et al., 2012). An independent t-test on the proportion of errors between the meditation and control group was significant, $t(42)=-$ 2.461, $p=0.018$.

\section{Visual proportion of errors}

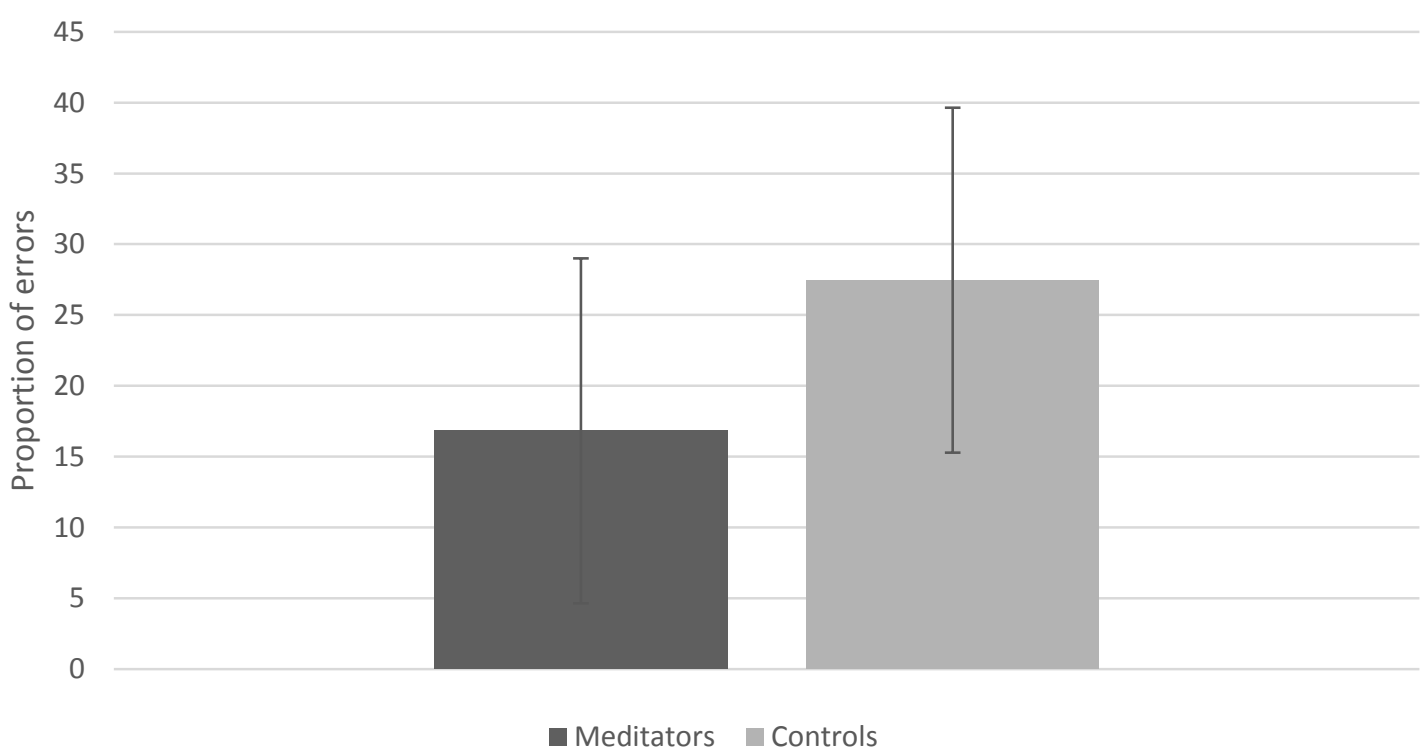

Figure 2. Visual experiment proportion of errors for Meditation and Control groups. 
The secondary interest in this study was whether response times differed between the groups. This is essential in reporting the performance on the SART as it is susceptible to speed-accuracy trade-offs. This means that low error rates might not necessarily indicate better performance as subjects may use strategy to respond slower in order to make fewer errors. Figure 3 shows the mean RTs for each response type. A 2 (group) by 3 (response type) mixed design ANOVA was conducted to determine if meditator and control groups differed in RT for each type of response. There was a main effect found for response type which indicated that there was a significant difference in RT between the types of responses (default, switch, error) $F(2,84)=17.47, p<0.01$. There was no main effect found for group $F(2,84)=0.78, p=0.46$ and no interaction $F(1,42)=0.31, p=0.58$. This means that meditators and controls did not differ in how long they took to respond and this was true for default, switch and error trials.

\section{Visual Response Times}

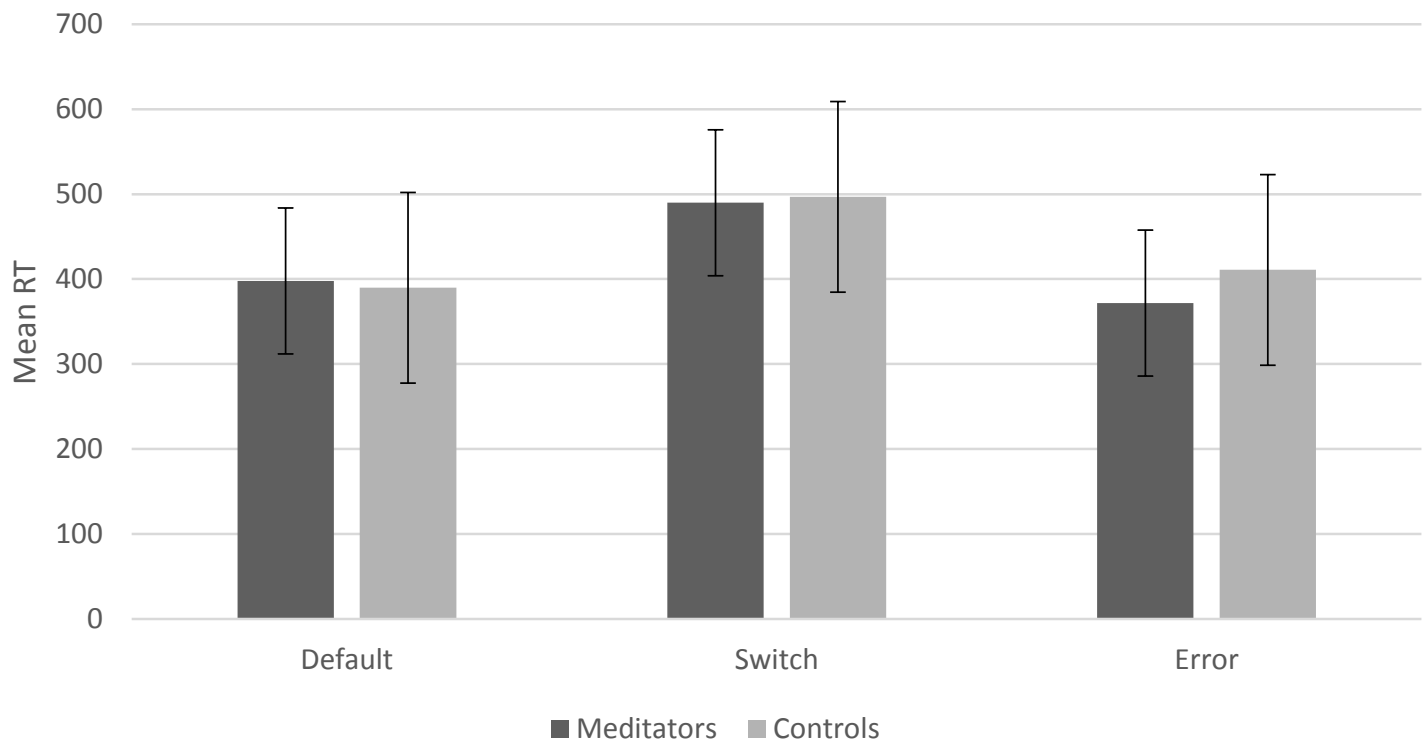

Figure 3. Visual experiment response times for Meditation and Control groups by response type. 
Planned comparisons were then conducted between response types across both groups. Subjects in the Cheyne and colleagues (2012) study were slowest when making a switch response and fastest when making an error response. Our results are consistent with their findings. As there was no significant interaction between group and response type, response times were collapsed across groups. Examination of the means showed that the switch RT was the slowest at 490ms ( $S D=90)$, followed by the default RT at 390ms seconds $(S D=70)$ and the error RT at 390ms $(S D=20)$. As three measures of RT were independently compared, a Bonferroni-adjusted significance level of 0.0167 was calculated to account for the increased possibility of type-I error. A Paired Samples t-test comparing RTs for trials with switch and default responses showed that switch responses had significantly slower RTs than default responses, $t(43)=8.157, p<0.01$. There was however no significant difference between the RTs for default and error responses, $t(43)=0.133, p=0.90$. Finally, there was a significant difference between the RTs for switch and error responses, $t(43)=4.258, p<$ 0.01. Overall these results were as expected, even though no significant effects were found between default and error responses. These indicate that individuals took longer to respond when they were required to inhibit their automatic response and switch responses for a target.

The next analyses were to determine if accuracy was dependent upon meditation experience within the meditation group. It was expected that more experienced meditators would have fewer errors than novice meditators. Correlations were completed between proportion of errors and frequency of meditation experience by years, sessions per week, minutes spent meditating per week, and minutes spent meditating per session. A significant negative correlation was found between the proportion of errors made and the frequency of years spent meditating, $r(20)=-.451, p=0.035$, more years of experience was associated with lower proportion of errors. This association is of moderate size (Cohen, 1988) where $20.3 \%$ of the variability in error scores can be explained by variability in meditation 
experience by years. No significant correlations were found between proportion of errors and sessions per week, $r(20)=0.21, p=0.927$, minutes spent meditating per week, $r(20)=$ $0.128, p=0.569$, minutes spent meditating per session, $r(20)=0.36, p=0.875$, and frequency of visits to meditation retreats, $r(20)=-0.21, p=0.359$.

Finally, it was examined whether the type of meditation practiced influenced the proportion of errors made. There was a lack of consistency in the self-reporting of type of meditation practiced with a vast range of different labels produced. Responses from the meditation questions were weighted so that a response of strongly agree/disagree was worth a score of 2 (or-2) and a response of neither agree or disagree was worth a score of 0 . These weighted scores were then subtracted to provide an index of -8 to +8 , where positive numbers would indicate a tendency and the extent to which someone practices FA and negative numbers indicate a tendency and extent to which someone practices OM. These scores were then correlated with the proportion of errors made. No significant differences in proportion of error were found between FA and OM meditators, $r(22)=-0.177, p=0.43$.

\section{Discussion}

The main findings from Experiment 1 were the significant difference for proportion of errors and the statistically similar RTs between meditators and non-meditators. Meditators made fewer errors than non-meditators and this superior performance was not due to a speedaccuracy trade off: meditators made their responses as quickly as the non-meditators. These results are consistent with previous studies that showed that meditators perform more accurately than non-meditators on other tasks that measure vigilance (Chambers, Lo, \& Allen, 2008; MacLean et al., 2010). Taken together, our results in combination with previous research indicates that the benefits to sustained attention through meditation are transferrable 
across multiple tasks measuring vigilance. This further demonstrates the validity of the SART as a measure of sustained attention.

The difference in errors made between meditators and controls has greater validity as this difference cannot be due to the groups using difference response strategies. As the SART is susceptible to speed-accuracy trade-offs, lower error rates could be due to subjects slowing responding in order to make fewer errors, rather than performing better at the task. However, since the groups did not differ in their response times across all trials, this shows that the difference in error is not due to a speed-accuracy trade-off and is therefore more likely to be due to the independent variable.

The performance by both meditators and the control group followed similar trends to previous studies that use the SART. The proportion of errors made by the control group is almost identical to the amount of errors made in the Cheyne and colleagues (2012) study, suggesting that the control group performed normally and were a good comparison for the meditation group. Therefore, any differences between the groups cannot be due to lack of motivation by the control group.

The examination of RT by response type showed that participants responded slowest on switch trials (where a target ' 3 ' appeared and subjects made a correct change in response), followed by default trials (a correct response to a non-target), and finally the fastest responding was for error trials (a failure to make a response change to a target ' 3 '). This coincides with previous studies (Cheyne et al., 2012), and indicates that participants were involved in an automatic responding when they made a target error, while they were involved in response inhibition when making a correct response to a target stimulus, thus taking more time to respond. 
While it was expected that more experienced meditators would have a lower proportion of errors made than less experienced meditators, this was not found for all measures of meditation experience level. There was only a significant correlation found between proportion of errors made and frequency of years spent meditating. While this coincides with previous research (van Vugt \& Slagter, 2014), it is unclear as to why no other significant correlations were found. It is likely that this was due to the small sample size of meditators. Furthermore, there was no significant difference in accuracy between FA and OM meditation. This could be due to limitations with the sample that did not allow for clearly defined groups. Many reports suggest that practice in FA and OM has much overlap and the two are not practiced exclusively, rather an individual must be well practiced in FA before learning OM (Kapleau, 2013). Another possibility is that the differences were masked by the level of meditation experience as previous studies have shown that a difference between FA and OM can only be observed in highly experienced meditators.

As these results support the argument that meditation has positive effects for sustained visual attention, it may be expected that meditation also has benefits for other modalities of sustained attention. While there is some research to suggest that meditation may affect auditory sustained attention, it is far less researched than visual vigilance. Therefore the next experiment was developed to examine whether people who practice meditation were more accurate at an auditory version of the SART than a control group of non-meditators.

\section{Experiment 2: Auditory}

\section{Method}

Participants. Majority of the same participants were recruited for the auditory task as for the visual task. Participants completed both tasks in a single session with a short break offered between. The order of completing the visual and auditory tasks was counterbalanced. 
Some of the subjects did not complete the visual task appropriately, which may indicate that they did not understand the task or stopped making responses part way through. Therefore, 7 subjects were excluded from the visual task while still being included in the auditory task. There were 25 meditation participants (11 males and 14 females with an average age of 37.96 years ranging from 18-69) and 26 control participants ( 8 males and 18 females with a mean age of 36 years ranging from 22-67).

Apparatus. The task was completed on the same Dell Precision T1650, 3.30GHz using the programming software Psychopy (Peirce, 2007). The stimuli were presented on a 23 inch $120 \mathrm{~Hz}$ Samsung LCD monitor in a private room. A chin rest was mounted on the table and was $60 \mathrm{~cm}$ away from the screen. Participants were required to wear headphones during this task.

Stimuli. The auditory version of the SART was similar to Experiment 1, except instead of visual stimuli, participants were presented with auditory stimuli. This consisted of 9 different tones ranging between $350-950 \mathrm{~Hz}$ in intervals of $75 \mathrm{~Hz}$ with the exception of the $650 \mathrm{~Hz}$ tone, which was replaced by a 'white noise' burst. This sound was chosen for its distinctness from the other tones, making it easy to identify in rapid succession. As in Experiment 1, the target stimuli were pseudo-randomly presented with an equal probability of $1,3,5$ or 7 non-target stimuli between each target. A target stimulus was never presented immediately after another target. The succession of trials was rapid with each stimulus only presented for $250 \mathrm{~ms}$ and each trial only lasted 1.15 seconds. No mask was used in this task as research has shown that auditory stimuli are less sensitive than visual to the subsequent presentation of stimuli (Potter, Chun, Banks \& Muckenhoupt, 1998). A total of 1000 trials were included, 200 of which were target trials, giving a proportion of $20 \%$ target trials.

Procedure. Participants were asked to sit comfortably in front of the computer with their head sitting in a chin rest. They were then asked to read the instructions on the computer screen which were reinforced with verbal instructions. Ample opportunity was given for 
questions to be asked. The task began with a set of ten practice trials. When participants felt comfortable with the task they could begin the experiment. Participants were instructed that speed was just as important as accuracy in this task, in that they should respond as quickly as they can and also as accurately as they can.

Participants performed an auditory version of the SART (Robertson et al., 1997). Figure 4 shows the general experimental paradigm. In a typical trial, subjects were presented with a fixation cross and a concurrently presented auditory stimulus (either target or nontarget) for 250ms. Participants were then required to make a response before the end of the trial which lasted $1150 \mathrm{~ms}$. The task lasted approximately 22 minutes and upon completion participants were given a debrief information sheet (APPENDIX D) and were verbally debriefed. Student control participants were given course credit and meditation (and nonstudent control) participants were given a movie voucher for their time.

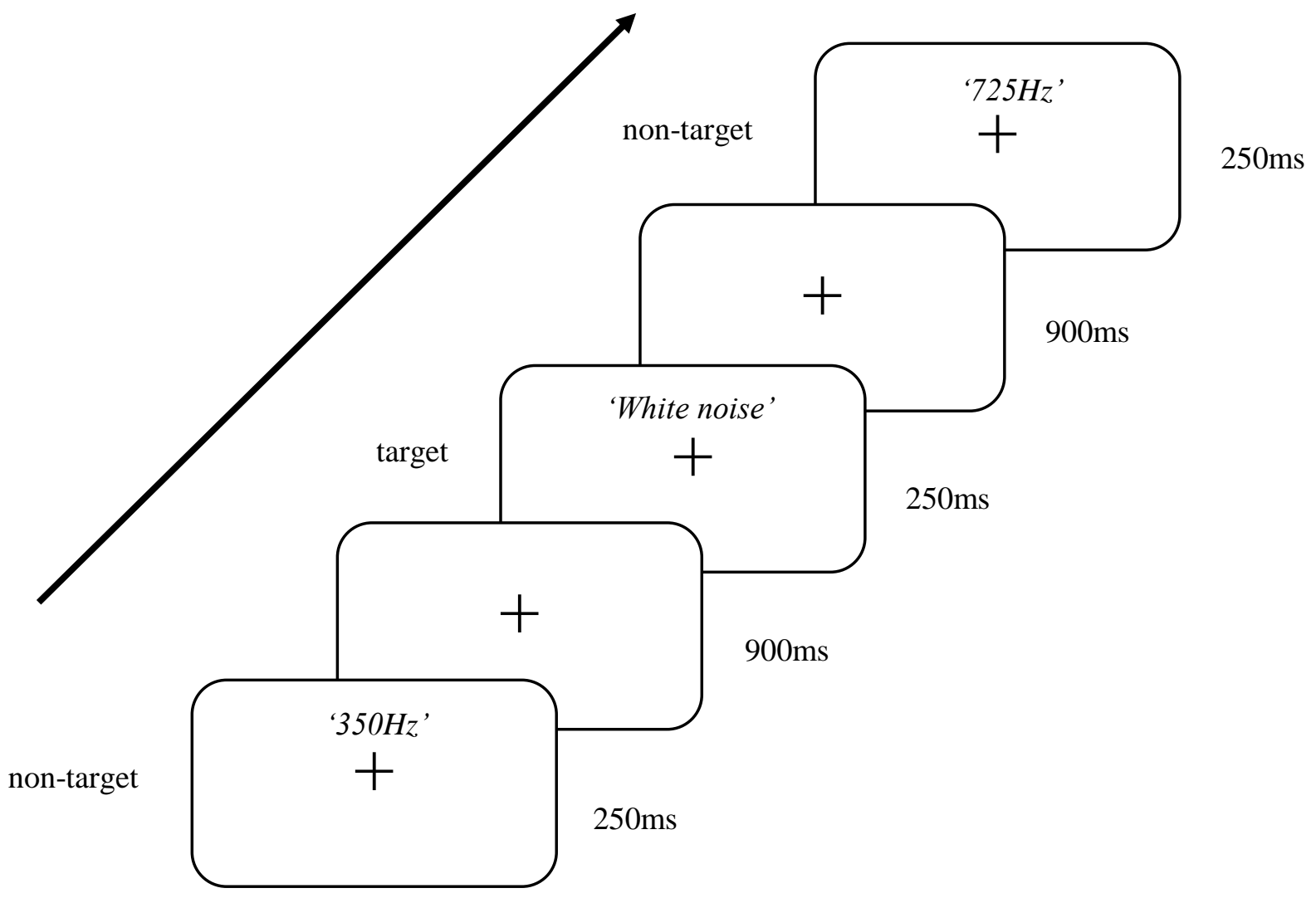

Figure 4. Experimental paradigm for Auditory Experiment. 
Responses. As in the visual task, participants responded to targets and non-targets by pressing either the left or right arrow. Half of the participants were instructed to press the left arrow key for non-targets and the right arrow key for targets and vice versa for the other half of participants. The assignment of the keys to stimuli was counterbalanced between participants.

Design. The design also remained the same as the visual task. A between-subject design was used with meditation experience (meditators vs. non-meditators) as the independent variable. The dependant variables include proportion of errors and four measures of reaction time; Default, Switch, Error and False alarm.

\section{Results}

Firstly, analyses were conducted to ensure that the meditation and control groups did not differ in terms of age, gender or level of education. The meditation group had a mean age of $37.96(S D=13.76)$ and the control group had a mean age of $36,(S D=11.16)$. An Independent T-test showed that the two groups did not differ significantly in terms of age, $t$ $(50)=.421, p=0.675$. The meditation group had more females than males (11 males and 14 females) as did the control group ( 8 males and 18 females). A chi-squared showed that the two groups did not significantly differ by gender, $\chi^{2}(1, N=49)=0.291, p=0.59$. Finally, education was examined by scoring participants self-reported highest level of education. As in the visual study education was rated from 1-4, from high school graduation to postgraduate study. A chi-squared showed that the two groups did not significantly differ by education, $\chi^{2}$ $(3, N=49)=2.587, p=0.46$.

The primary interest of the auditory vigilance study was to examine a difference in accuracy between meditators and non-meditators. The average frequency of errors in the meditation group was $14.6(S D=12.25)$ and in the control group was $26.07(S D=19.14)$. The 
mean frequency of false alarm errors were very low overall; $2.72(S D=2.82)$ and $3.42(S D=$ 3.15) for meditators and non-meditators, respectively. Therefore, as in Experiment 1 and previous SART studies (Cheyne et al., 2012), false alarms will not be included in any further analyses.

Overall, the mean proportion of errors made (see Figure 5) was greater for the control group, $12.73 \%(S D=9.57)$, than the meditation group, $7.3 \%(S D=6.13)$. This was confirmed by a significant result from conducting an independent t-test: $t(50)=-2.02, p=$ 0.048. Even without statistically comparing the proportion of errors between the visual and auditory experiments, it is observable that the proportion of errors is noticeably smaller in the auditory experiment. This level of performance in this auditory task is consistent with other SART experiments that also used auditory stimuli (Seli et al., 2012).

\section{Auditory proportion of errors}

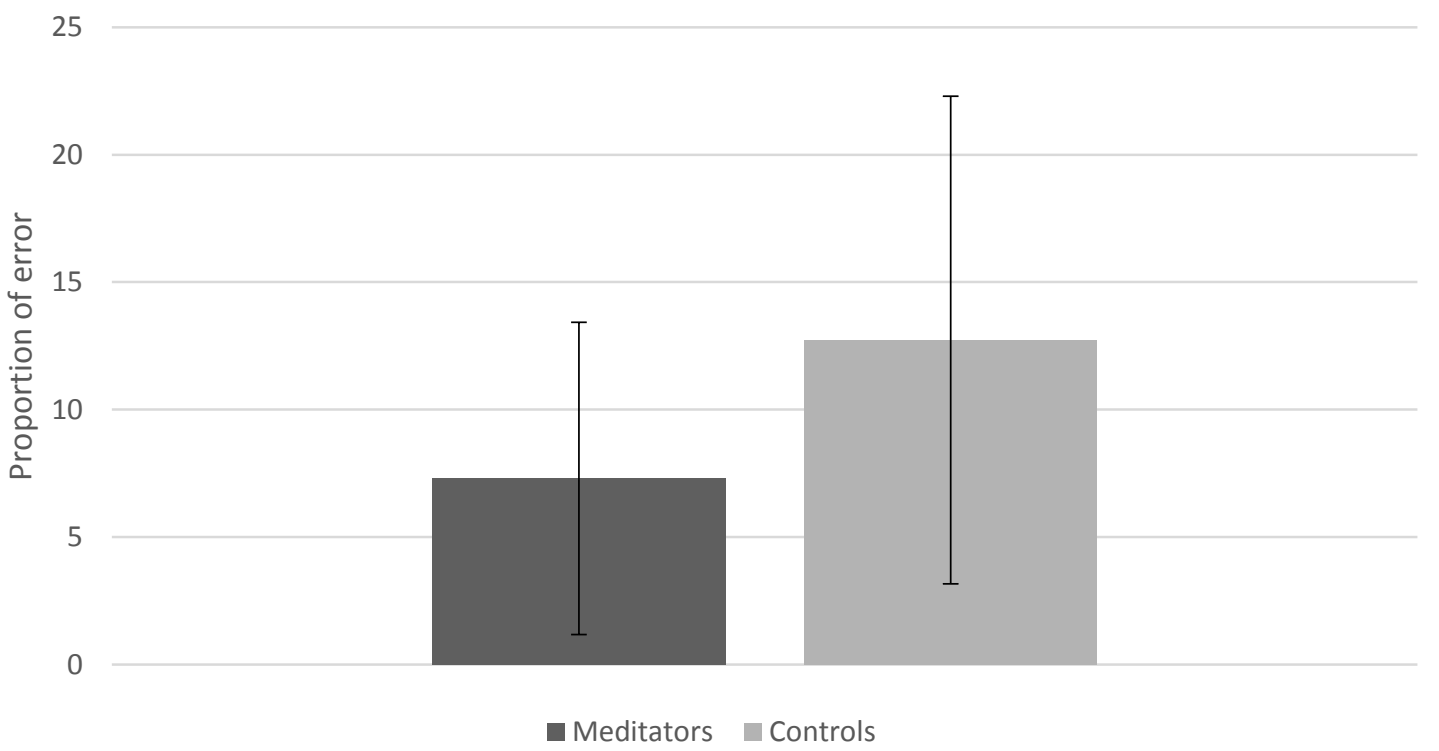

Figure 5. Auditory experiment proportion of errors for Meditation and Control groups.

A 2 (group) by 3 (response type) mixed design ANOVA was conducted to determine if the meditator and control groups differed in RT for each response. Figure 6 shows the 
mean RTs for each response type. As with the visual task, there was a main effect found for response type, $F(2,98)=14.54, p<0.01$, which indicates that overall there was a significant difference in RT between the types of responses (default, switch, error). Also there was a main effect found for group, $F(1,49)=8.84, p=0.005$, which indicates that there was a significant difference between groups. Across all responses, the average RT for the meditation group was 551ms $(S D=117)$ and $492 \mathrm{~ms}$ for the control group $(S D=233)$, indicating that the meditation group performed significantly slower. There was no significant interaction found between group and response type, $F(2,98)=0.09, p=0.91$.

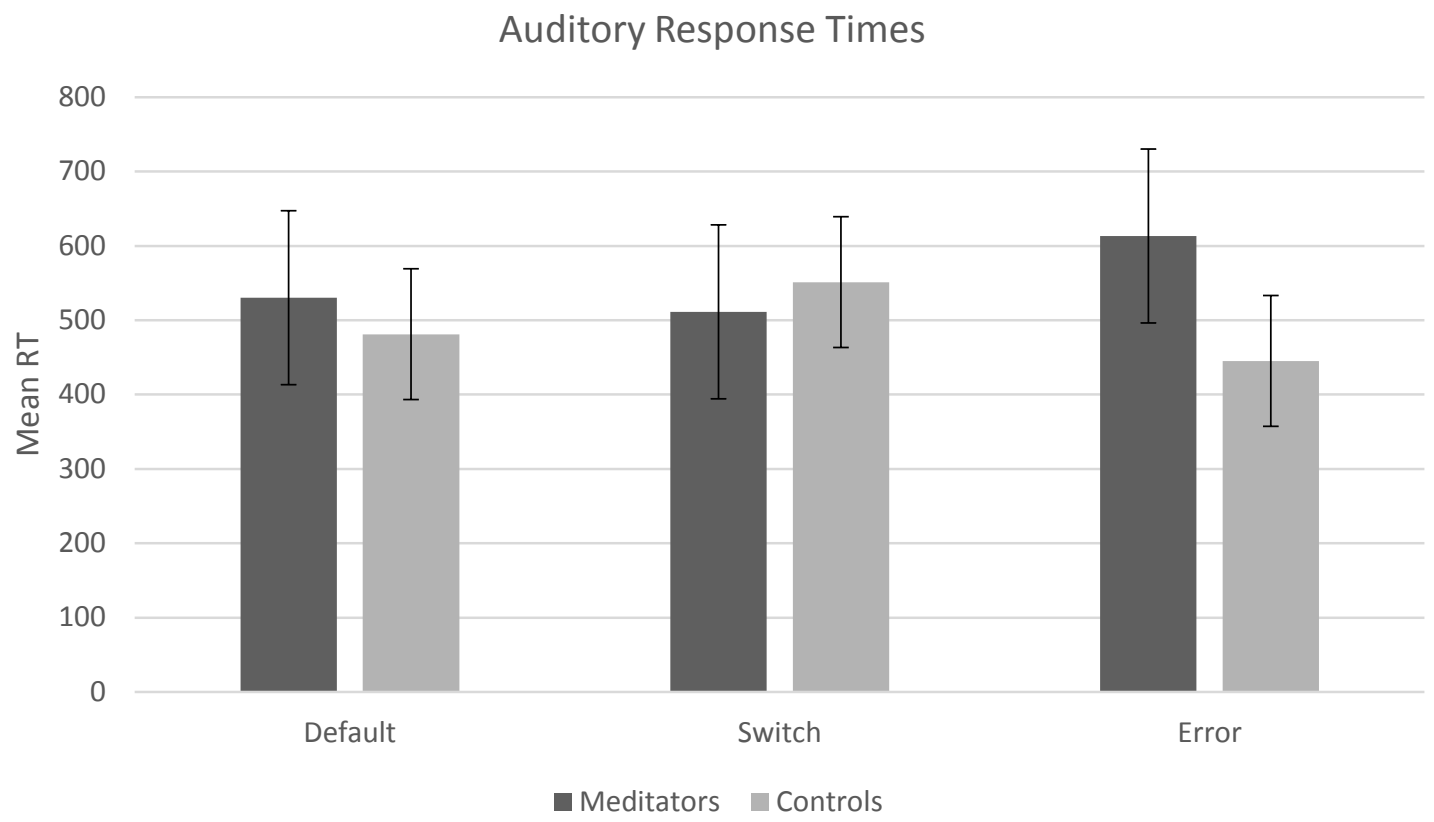

Figure 6. Auditory experiment response times for Meditation and Control groups by response type.

As the meditation group responded significantly slower than the control group, analyses were completed to determine whether meditators used a response strategy in order to increase accuracy in the task while inadvertently sacrificing speed. There was no significant correlation found between accuracy and default RT, $r(23)=-0.3286, p=0.117$. These results show that even though the mediation group responded more slowly, this had no significant 
influence on accuracy; therefore the speed-accuracy trade-off did not artificially inflate accuracy scores.

Planned comparisons were then conducted between response types. Again, it was expected that switch responses would be the slowest and error responses would be the fastest (Cheyne et al., 2012). As there was no significant interaction between group and response type, response times were collapsed across groups. Examination of the means showed that the switch RT was the slowest at $580 \mathrm{~ms}(S D=96)$, followed by the default RT at $500 \mathrm{~ms}(S D=$ $82)$ and the error RT at $480 \mathrm{~ms}(S D=147)$. As three measures of RT were independently compared, a Bonferroni-adjusted significance level of 0.0167 was calculated to account for the increased possibility of type-I error. A Paired Samples t-test comparing RTs for switch and default trials showed that switch trials had significantly slower RTs than default trials, $t$ $(51)=6.56, p<0.01$, and error responses, $t(51)=4.48, \mathrm{p}<0.01$. There was, however, no significant difference between the RTs for default and error trials, $t(51)=1.24, p=0.22$. Overall, these results were as expected, even though no significant effects were found between default and error trials. These indicate that individuals took longer to respond when they were required to inhibit their automatic response and switch responses for a target trial.

The next set of analyses set to determine whether accuracy on the SART was affected by meditation experience. Correlations were completed between proportion of errors and frequency of meditation experience by years, sessions per week, minutes spent meditating per week, minutes spent meditating per session, and frequency of visits to meditation retreats. There were no significant correlation found between the proportion of errors made and the frequency of years spent meditating, $r(24)=-0.109, p=0.596$, but the correlations were trending toward significance between proportion of errors and sessions per week, $r(24)=-$ $0.358, p=0.072$, and between proportion of errors and minutes spent meditating per week, $r$ $(24)=-0.335, p=0.094$. However, there was a significant negative correlation found between 
proportion of error and minutes spent meditating per session, $r(24)=-0.441, p=0.02$. This association was of moderate size (Cohen, 1988) where $19.4 \%$ of the variability in error scores can be explained by variability in meditation experience by minutes spent meditating per week.

Finally, analyses were conducted to determine whether the type of meditation practiced influenced the proportion of errors made. As in the visual experiment the responses from the meditation questions were scored along an index, where positive number would indicate being closely aligned with FA and negative numbers indicate being more closely aligned with OM. These scores were then correlated with the proportion of errors made. No significant differences in proportion of error were found between FA and OM meditators, $r$ $(24)=-0.116, p=0.573$.

\section{Discussion}

The results from the auditory version of the SART were overall consistent with the hypotheses and previous research. The group which practiced meditation made significantly less errors than the control group when the target 'white noise' was presented. This mimics the results from the visual experiment and is consistent with previous studies that showed that meditators perform more accurately than non-meditators on tasks that measure auditory vigilance (Lutz et al., 2009; Valentine \& Sweet, 1999).

Overall the proportion of errors made are observably fewer than the original visual SART, while response times were slower. These results are similar to Seli and colleagues (2012) study that developed an auditory version of the SART and found greater accuracy but slower RTs. This suggests that these factors are a product of the task and likely do not reflect the subjects performance. It also suggests that the subjects are processing the auditory stimuli differently than the visual stimuli. Finally, the similarities between this experiment and the 
results found in the study by Seli and colleagues (2012) also further demonstrate the validity of the SART as a measure of auditory sustained attention.

The RT results are similar to those in Experiment 1. Switch responses (making a correct response to a target) were significantly slower than other responses, indicating that subjects were required to inhibit their automatic response in order to make a correct change which slowed their responding. While the default and error responses were faster than switch responses, they did not significantly differ. This means that subjects were just as fast to respond when they correctly responded to a non-target tone as when they incorrectly responded to the target 'white noise'. This is somewhat inconsistent with previous reports of RTs by response as Cheyne and colleagues (2012) found that error responses were faster than default responses. However, these results still show that subjects were engaged in automatic or 'mindless' responding during both default and error responses.

There was a difference found between groups in overall RT where meditators responded slower than the control group. These results were contrary to hypotheses which expected both groups to respond at the same speed. Both groups were given identical instructions that speed was just as important as accuracy; to respond as fast but also as accurately as possible. However, as there were no significant correlations found between RT and error, this suggests that there was no speed-accuracy trade-off taking place. The greater accuracy by meditators was not artificially inflated by a speed-accuracy trade-off as there was no association between accuracy and speed in this task. Therefore meditation subjects performed more accurately at the task regardless of speed.

While it was expected that more experienced meditators would have a lower proportion of errors made than less experienced meditators, this was not found for all measures of meditation experience level. There was only a significant negative correlation 
found between proportion of errors made and minutes spent meditating per session. This is unexpected as the only correlation found in the visual study was between proportion of errors made and frequency of years spent meditating. These findings together suggest that there is a complex relationship between accuracy in vigilance tasks and meditation experience. This is supported by previous research which has shown a connection between meditation experience and accuracy at vigilance tasks (van Vugt \& Slagter, 2014). It is unclear as to why no other significant correlations were found in this study; however, it may be due to the small sample size of meditators which may have masked any other significant results. Furthermore, as with the visual experiment there was no significant difference found between FA and OM meditation. As mentioned, this could be due to limitations with the sample that did not allow for clearly defined groups or that the differences were masked by the level of meditation experience.

As there were significant differences between meditators and non-meditators in the accuracy of both visual and auditory versions of the SART, it could be expected that this would also occur when both visual and auditory stimuli are combined into the same task. There is very little research on crossmodal vigilance studies and no previous study that examines this in regards to meditation. Therefore, the next experiment was developed to examine whether people who practice meditation were more accurate at two crossmodal versions of the SART than a control group of non-meditators. 


\section{Experiment 3A: Auditory Target Visual Non-target}

\section{Method}

Participants. Twenty-two experienced meditators (11 males and 11 females) with a mean age of 35.5 (age range 20-55 years) were recruited as our meditation group. Twentythree participants with no meditation experience (10 males and 13 females) with a mean age of 32.5 (age range of 20-52 years) made up our control group. All participants had normal or corrected vision and hearing. Participants were offered a movie voucher for their participation. All participants received detailed information regarding the study (APPENDIX A) and gave written consent (APPENDIX B) before beginning the experiment.

Apparatus. The task was completed on the same Dell Precision T1650, 3.30GHz using the programming software Psychopy (Peirce, 2007). The stimuli were presented on a 23 inch $120 \mathrm{~Hz}$ Samsung LCD monitor in a private room. A chin rest was mounted on the table and was $60 \mathrm{~cm}$ away from the screen. Participants were required to wear headphones during this task.

Stimuli. This task included auditory target stimuli and visual non-target stimuli. The visual non-target were single digits that could be any number from 1 to 9 , randomly determined. An infrequent auditory target stimulus was pseudo-randomly presented at various times throughout the task. This target was a static 'white noise' that was used as the target in the auditory experiment. This was accompanied by a fixation cross. As in the previous experiments, there was an equal probability of $1,3,5$ or 7 non-target stimuli between each target. A target stimulus was never presented immediately after another target. A total of 1000 trials were included, 200 of which were target trials, giving a proportion of $20 \%$ target trials. 
Procedure. The procedure remained the same as the other two experiments.

Participants performed a crossmodal version of the SART (Robertson et al., 1997) with auditory target stimuli and visual non-target stimuli. Figure 7 shows the general experimental paradigm. In a non-target trial participants were presented with a visual stimulus (number 19) for $250 \mathrm{~ms}$. This was followed by a mask ('\&' symbol) for $900 \mathrm{~ms}$. Participants were required to make a key response before the end of the trial (lasting 1150ms). During a target trial an auditory stimulus (white noise burst) was presented for $250 \mathrm{~ms}$. A fixation cross was concurrently presented. Participants were required to make a key response before the end of the trial (lasting $1150 \mathrm{~ms}$ ). The task lasted approximately 22minutes and upon completion participants were offered an ample break before beginning the next task.

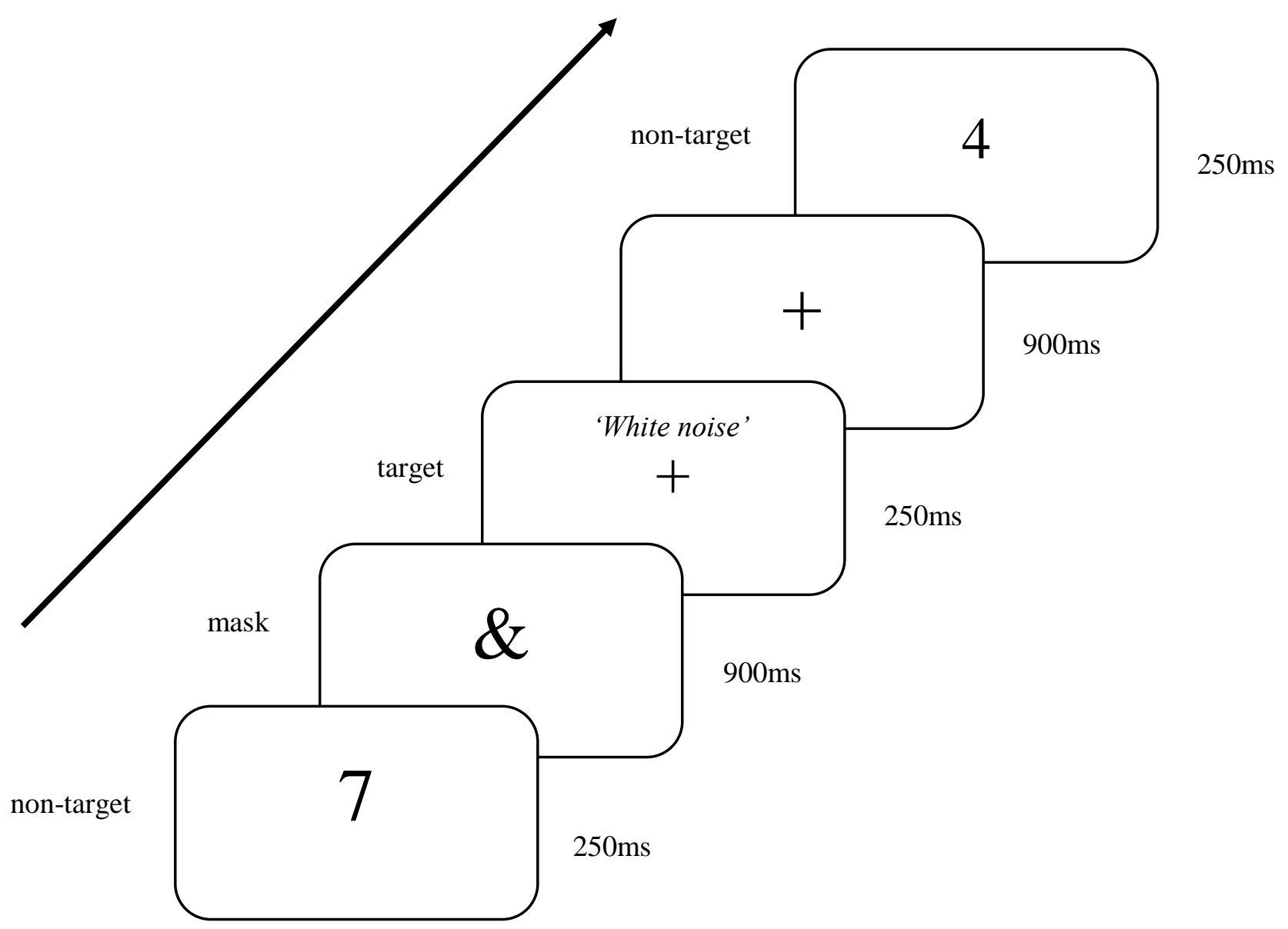

Figure 7. Experimental paradigm for Auditory-target experiment. 
Responses. Participants responded to targets and non-targets by pressing either the left or right arrow key. Half of the participants were instructed to press the left arrow key for non-targets and the right arrow key for targets and vice versa for the other half of participants. The assignment of the keys to stimuli was counterbalanced between participants.

Design. The design remained the same as the two previous experiments; a betweensubject design with meditation experience (meditators vs. non-meditators) as the independent variable. Again the primary dependant variable of interest was the proportion of errors made. Four measures of reaction time were included which represented the four possible responses (as per Cheyne et al., 2012).

\section{Results}

As this group of participants differed to the previous experiments, analyses were conducted to determine that the meditation and control group did not differ in terms of age, gender or level of education. The meditation group had a mean age of $35.45(S D=11.54)$ and the control group had a mean age of 32.57, $(S D=10.92)$. An Independent T-test showed that the two groups did not differ significantly in terms of age, $t(43)=-.863, p=0.75$. The meditation group had an equal number of females and males (11 males and 11 females) while the control group had slightly more females than males (10 males and 13 females). A chisquared showed that the two groups did not significantly differ by gender, $\chi^{2}(1, N=45)=$ $0.192, p=0.661$. Finally education was examined by scoring participants self-reported highest level of education. Education was rated from 1-4, from high school graduation to postgraduate study. A chi-squared showed that the two groups did not significantly differ by gender, $\chi^{2}(3, N=45)=0.355, p=0.949$.

This experiment focused on examining differences in performance between meditators and non-meditators on a crossmodal version of the SART that included auditory 
targets and visual non-targets. The meditation group had a mean of $30.36(S D=29.93)$ target errors, where the target appeared on the screen and the subject failed to switch responses. In comparison the control group had a mean of $54.09(S D=35.75)$ target errors. The mean frequency of false alarm errors were very low overall; $5(S D=6.94)$ and $3.78(S D=4.96)$ for meditators and non-meditators, respectively. Therefore, as with the previous experiments false alarms will not be included in any further analyses.

The mean proportion of errors made was fewer in the meditation group, $15.18 \%(S D=$ 14.97), than the control group, $27.04 \%$ ( $S D=17.87)$. This can be seen in Figure 8 . An independent $t$-test was conducted and showed that meditators made significantly less errors than non-meditators, $t(43)=2.408, p=0.02$.

\section{Auditory-target proportion of errors}

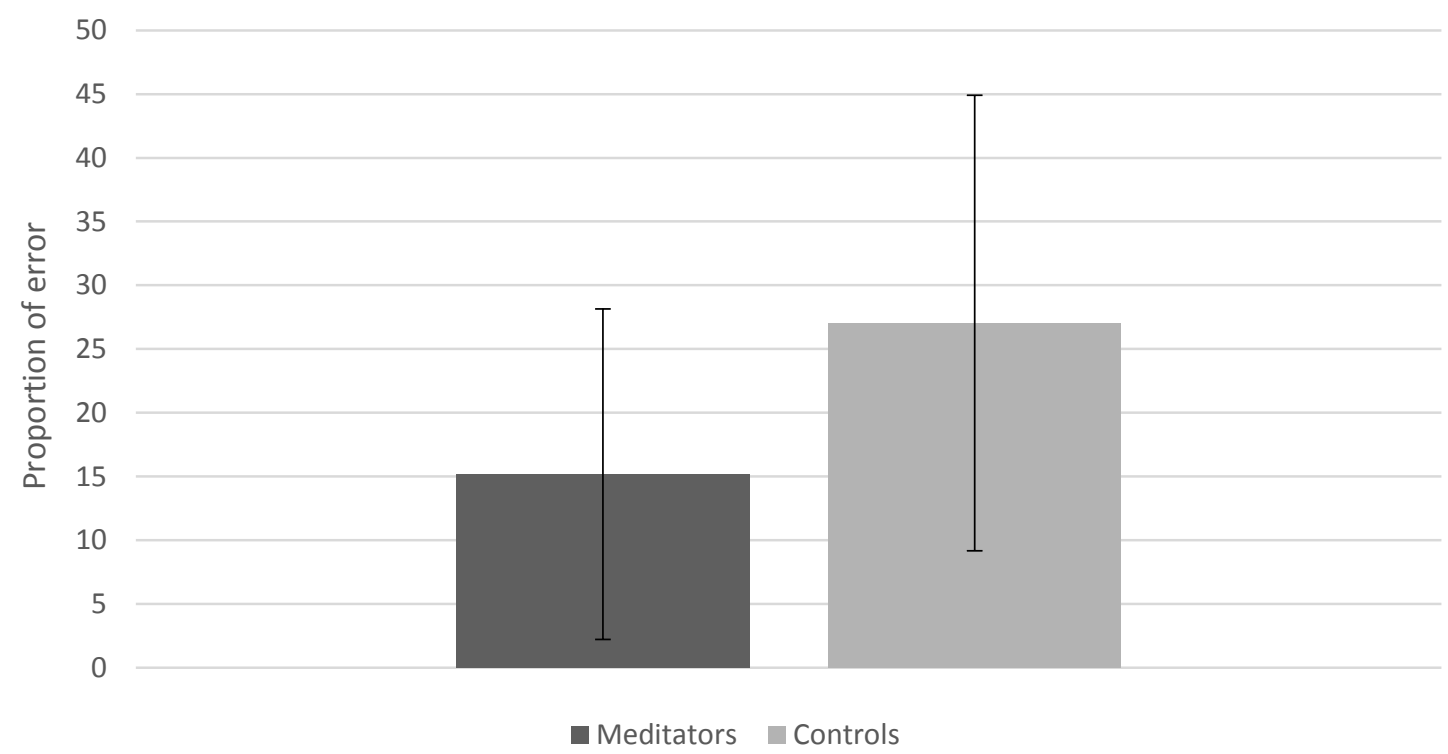

Figure 8. Auditory-target experiment proportion of errors for Meditation and Control groups.

The next analyses were to determine whether the response times differed between groups. A 2 (group) by 3 (response type) mixed design ANOVA was conducted to determine if meditator and control groups differed in RT for each type of response. Figure 9 shows the 
mean RTs for each response type. As with the previous tasks, there was a main effect found for response type, $F(2,84)=14.02, p<0.01$, which indicates that overall there was a significant difference in RT between the types of responses (default, switch, error). There was also a main effect found for group, $F(1,42)=5.34, p=0.026$, which indicates that there was a significant difference in meditators and controls across all responses. However, there was no significant interaction found, $F(2,84)=0.487, p=0.62$. This means that meditators and controls did not differ in how long they took to respond for default, switch and error trials.

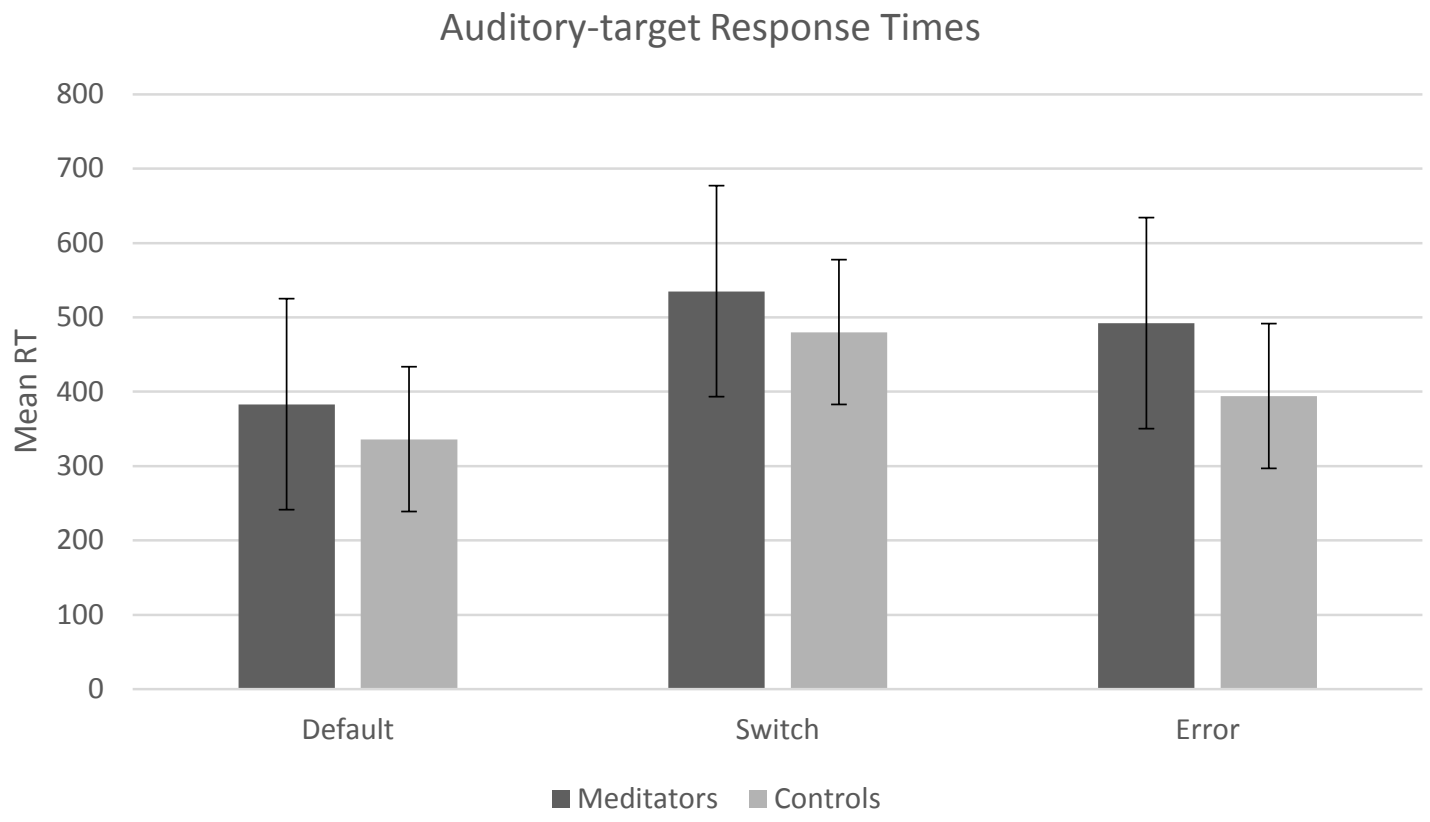

Figure 9. Auditory-target experiment response times for Meditation and Control groups by response type.

As the meditation group responded significantly slower than the control group, analyses were completed to determine whether meditators used a response strategy in order to increase accuracy in the task while inadvertently sacrificing speed. There was a significant correlation found between accuracy and default $\mathrm{RT}, r(21)=-0.593, p=0.004$. These results show that meditators were responding significantly slower than controls; therefore a speedaccuracy trade-off may have artificially inflated accuracy scores. 
Following up on the main effect for response type, post hoc comparisons were performed to examine the differences between response types. Examination of the means showed that the switch RT was the slowest at $510 \mathrm{~ms}(S D=0.08)$, followed by the error RT at $440 \mathrm{~ms}(S D=0.23)$ and then the default RT at $360 \mathrm{~ms}(S D=0.06)$. As three measures of RT were independently compared, a Bonferroni-adjusted significance level of 0.0167 was calculated to account for the increased possibility of type-I error. A Paired Samples t-test comparing RTs for switch and default trials showed that switch trials had significantly slower RTs than default trials, $t(44)=18.14, p<0.01$. There was also a significant difference between the RTs for default and error trials, $t(44)=-2.416, p=0.02$, where default trial RTs were significantly slower than error trial RTs. However there was no significant difference between the RTs for switch trials and error trials, $t(44)=1.976, p=0.55$. These results were not as expected, as default trials were significantly faster than error trials and yet error trials did not significantly differ from switch trials.

The next analyses set to determine whether accuracy on the SART was affected by meditation experience. Correlations were completed between proportion of errors and frequency of meditation experience by years, sessions per week, minutes spent meditating per week, minutes spent meditating per session, and frequency of visits to meditation retreats. There were no significant correlations found between the proportion of errors made and; the frequency of years spent meditating, $r(21)=-0.085, p=0.71$, minutes spent meditating per week, $r(21)=-0.237, p=0.3$, minutes spent meditating per session, $r(21)=-0.04, p=0.86$, or frequency of visits to meditation retreats, $r(21)=-0.384, p=0.09$, although this was trending toward significance. There was however a significant negative correlation between the proportion of errors made and the frequency of times spent meditating per week, $r(21)=$ $-0.444, p=0.044$ 
Finally, we looked at whether meditators more associated with a FA type of meditation were more accurate at the task then meditators associated with an OM type of meditation. As in the previous experiments, the responses from the meditation questions were scored along an index and were correlated with the proportion of errors made. No significant differences in proportion of error were found between FA and OM meditators, $r(21)=-$ $0.082, p=0.717$.

\section{Experiment 3B: Visual Target Auditory Non-target}

\section{Method}

Participants. The same participants from experiment 3A participated in this experiment. This included twenty-two experienced meditators (11 males and 11 females) with a mean age of 35.5 (age range 20-55 years), and twenty-three participants with no meditation experience (10 males and 13 females) with a mean age of 32.5 (age range of 2052 years).

Apparatus. The task was completed on the same Dell Precision T1650, 3.30GHz using the programming software Psychopy (Peirce, 2007). The stimuli were presented on a 23 inch $120 \mathrm{~Hz}$ Samsung LCD monitor in a private room. A chin rest was mounted on the table and was $60 \mathrm{~cm}$ away from the screen. Participants were required to wear headphones during this task.

Stimuli. In the Visual Target task, the auditory non-targets were tones ranging in frequency from $350-950 \mathrm{~Hz}$ in intervals of $75 \mathrm{~Hz}$, randomly determined. The visual target was the same used in Experiment 1; the presentation of the number ' 3 '. All other stimulus parameters (timing and probability of presentation) were identical to our other tasks.

Procedure. The general procedure was the same as the other experiments. Participants completed a crossmodal version of the SART (Robertson et al., 1997) with visual 
target stimuli and auditory non-target stimuli. Figure 10 shows the general experimental paradigm. In a non-target trial participants were presented with an auditory stimulus (tones $350-950 \mathrm{~Hz}$ ) for $250 \mathrm{~ms}$. A fixation cross was concurrently presented until the end of the trial. Participants were required to make a key response before the end of the trial (lasting $1150 \mathrm{~ms}$ ). During a target trial a visual stimulus ('3’) was presented for $250 \mathrm{~ms}$ and again participants were required to make a key response before the end of the trial (lasting $1150 \mathrm{~ms})$. The task lasted approximately 22 minutes and upon completion participants were offered an ample break before beginning the next task.

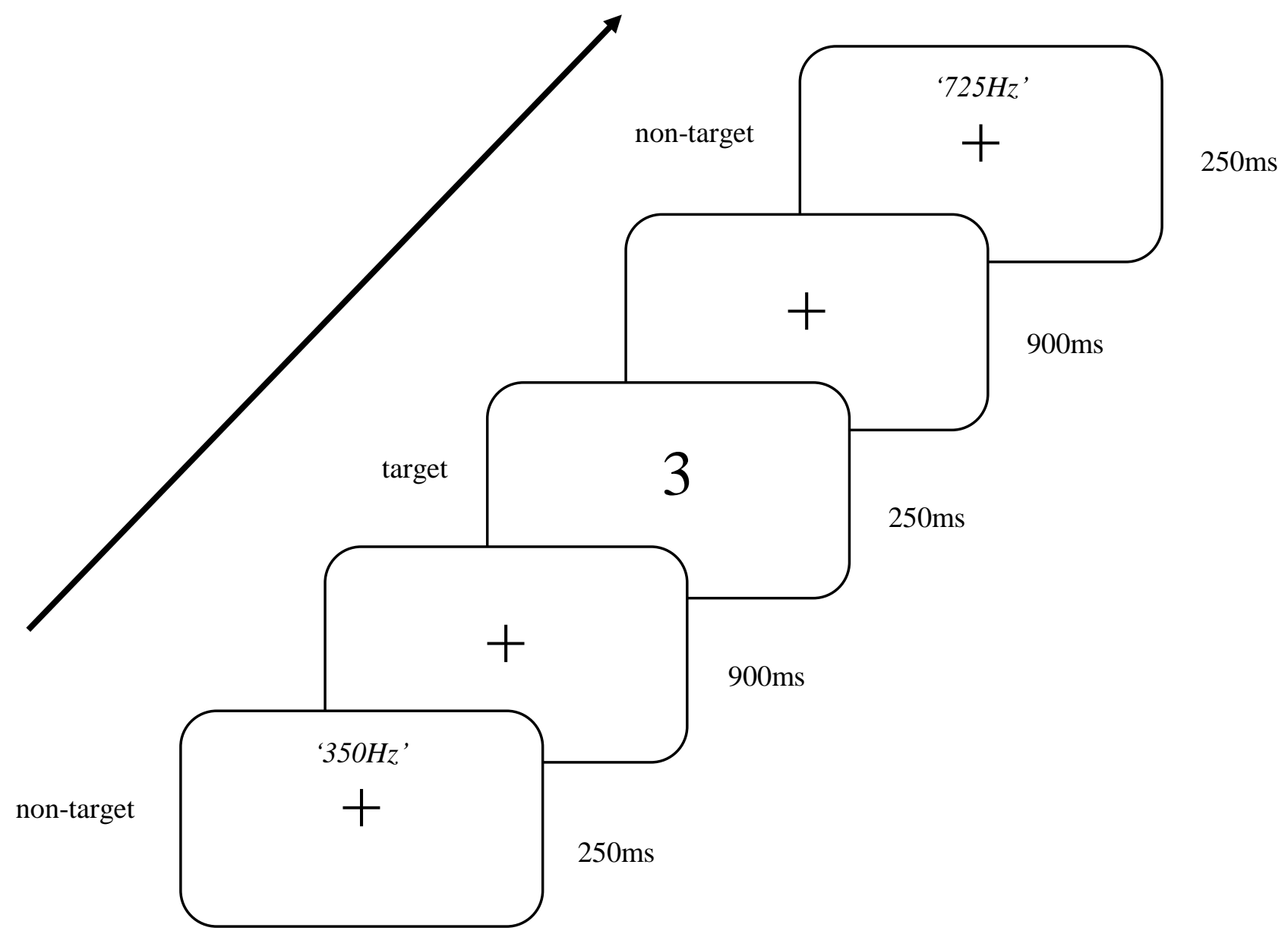

Figure 10. Experimental paradigm for Visual-target experiment. 
Responses. Participants responded to targets and non-targets by pressing either the left or right arrow key. Half of the participants were instructed to press the left arrow key for non-targets and the right arrow key for targets and vice versa for the other half of participants. The assignment of the keys to stimuli was counterbalanced between participants.

\section{Results}

In the Visual Target task, the meditators had a mean of $9.91(S D=15.87)$ target errors, while the control group made $23.87(S D=22.31)$ target errors. As with all tasks so far the frequency of false alarm errors were very low; $1.5(S D=2.06)$ for meditators and $2.7(S D=$ 3.51) for non-meditators. As with previous experiments the false alarms will not be analysed further. The mean proportion of errors made was greater for the control group, $11.93 \%$ ( $S D=$ 9.16), than for the meditation group, 4.95\% (SD=2.93) (see Figure 11). An independent $t$-test was conducted and showed that meditators made significantly less errors than nonmeditators, $t(43)=2.409, p=0.02$.

\section{Visual-target proportion of errors}

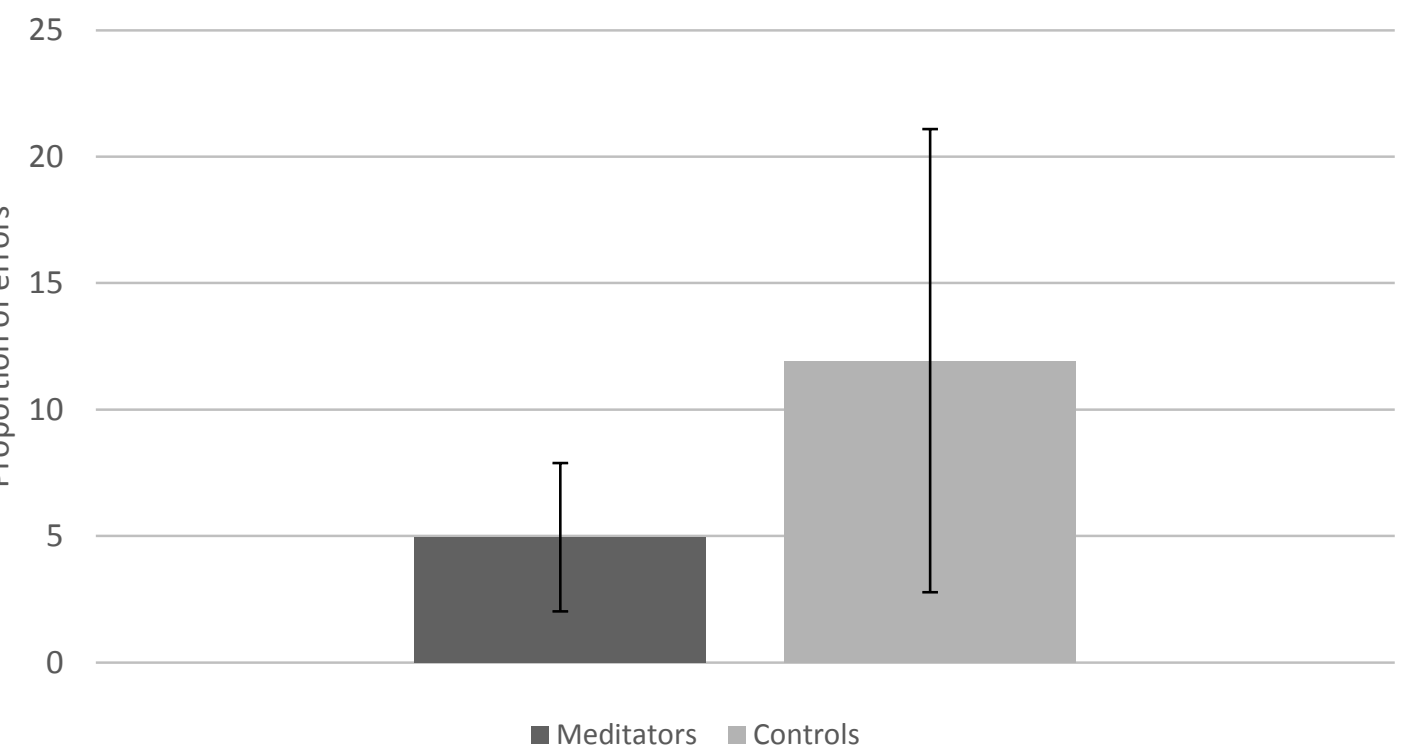

Figure 11. Visual-target experiment proportion of errors for Meditation and Control groups. 
The next analyses were to determine whether the response times differed between groups. A 2 (group) by 3 (response type) mixed design ANOVA was conducted to determine if meditator and control groups differed in RT for each response. Figure 12 shows the mean RTs for each response type Unlike the previous tasks, there was no main effect found for response type, $F(2,72)=.425, p=0.656$, no main effect found for group, $F(1,36)=0.903$, $p=0.348$, and no significant interaction found, $F(2,72)=0.001, p=0.999$. This means that the meditation and control group did not significantly differ from each other in RTs, and the switch, default and error trials did not differ in RTs.

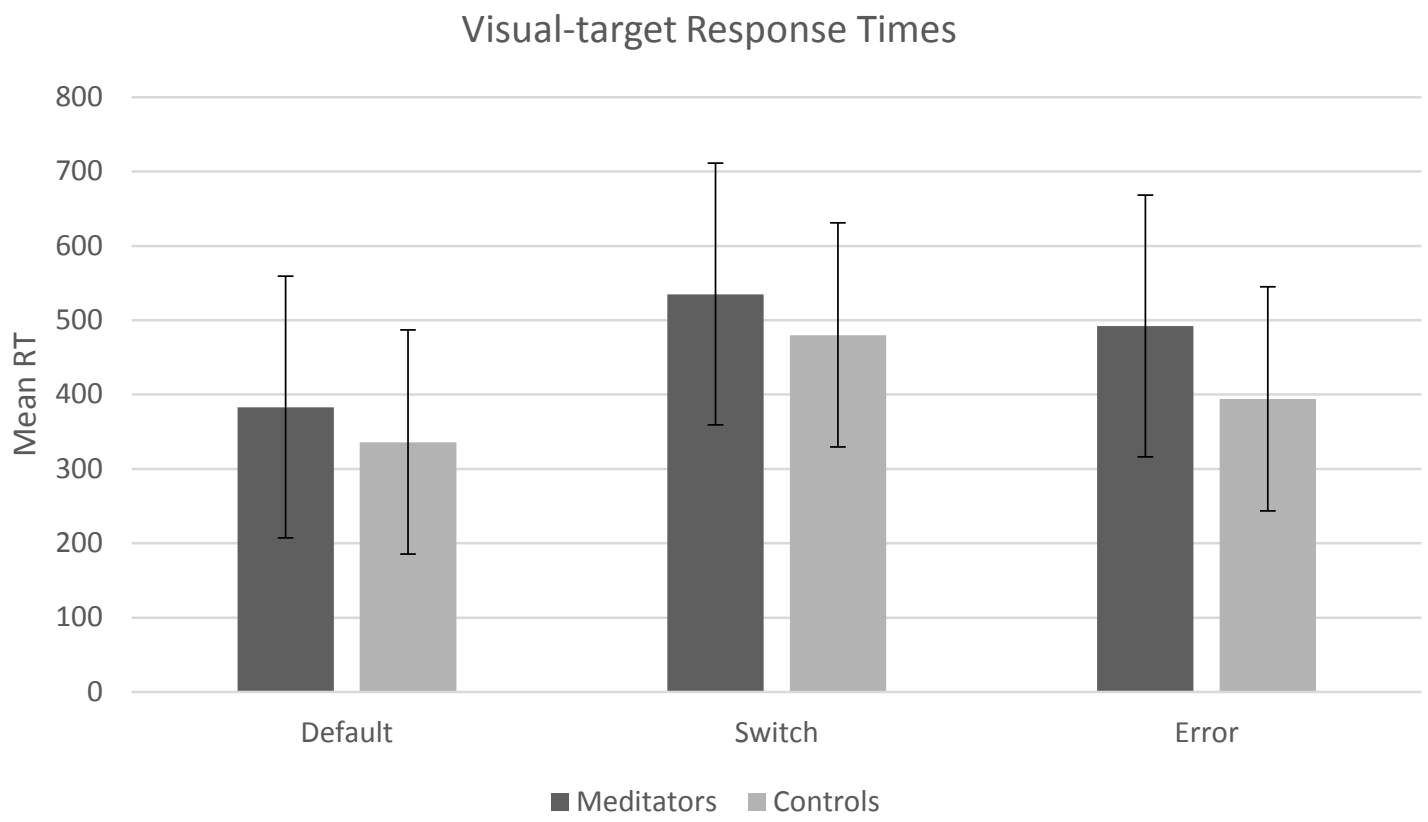

Figure 12. Visual-target experiment response times for Meditation and Control groups by response type.

The next analyses aimed to determine whether accuracy on the SART was affected by meditation experience. Correlations were completed between proportion of errors and frequency of meditation experience by years, sessions per week, minutes spent meditating per week, minutes spent meditating per session, and frequency of visits to meditation retreats. There were no significant correlations found for any of the variables. This included between 
the proportion of errors made and; the frequency of years spent meditating, $r(21)=0.017, p=$ 0.939, the frequency of times spent meditating per week, $r(21)=-0.292, p=0.187$, minutes spent meditating per week, $r(21)=-0.161, p=0.474$, minutes spent meditating per session, $r$ $(21)=0.259, p=0.245$, and frequency of visits to meditation retreats, $r(21)=-0.093, p=$ 0.680 .

Finally, we looked at whether meditators more associated with a concentrative type of meditation were more accurate at the task then meditators associated with a mindfulness type of meditation. The scores from the meditation index were then correlated with the proportion of errors made. No significant differences in proportion of error were found between FA and OM meditators, $r(21)=-0.257, p=0.248$.

\section{Discussion}

The results from the two crossmodal versions of the SART were overall consistent with the hypotheses, in that the group which practiced meditation made significantly less errors than the control group when the targets (either visual or auditory) were presented. These results are consistent with the results from the visual and auditory experiments and suggest that meditation has benefits to sustained attention across modalities rather than just independently within visual and auditory domains. This has implications for the way in which attention is conceptualised.

Overall, the Visual Target-Auditory Non-target task in Experiment 3B showed similar results as the auditory SART task in Experiment 2 as there was an overall lower proportion of errors and slower RT. The Auditory Target-Visual Non-target task in Experiment 3A showed similar results as the visual SART task in Experiment 1. In a previous study that developed a crossmodal version of the SART, they found that the proportion of errors in the crossmodal task were lower than the unimodal visual SART and higher than the unimodal auditory SART 
(Seli et al., 2012). It is important to note that in their crossmodal task visual and auditory stimuli were presented concurrently, and therefore, only included one crossmodal task. It is also worth mentioning that while they found a slowing in overall RT for the auditory targets compared to the crossmodal and visual tasks like in the present study, RT variability was not significantly different across all tasks. As RT variability is sensitive to changes in RT such as speeding and slowing due to lapses of attention, it suggests that rates of attentional lapses were consistent across all tasks. While RT variability was not looked at in this study, the results of the Seli and colleagues (2012) study give indication that the visual, auditory and crossmodal tasks may be comparable in terms of how they measure vigilance and attention lapses.

The RTs by response type did not coincide with previous studies in either of the crossmodal tasks. In the Auditory Target task there was a difference between switch and default trials, yet no significant difference between switch trials and error trials. This means that subjects did not respond any slower when they had to inhibit their automatic response in order to respond correctly for a target, than when they automatically made an incorrect response to a target. In the Visual Target task there was no difference in RT between any of the response types. This could mean that subjects did not engage in automatic responding during the frequent non-target stimuli. It could also suggest that subjects responded so slowly that any response inhibition to target trials was masked.

There was no difference in RT between meditators and the control group in Visual Target task. This was as expected and suggests that while meditators were more accurate at the task they were not engaging in any response strategies. Therefore, the results cannot be explained by a speed-accuracy trade-off. However, there was a significant difference found between meditators and the control group in the Auditory Target task, where meditators responded slower. As with the unimodal auditory task in Experiment 2, this was contrary to 
the hypothesis which expected both groups to respond at the same speed. As there was a significant correlation found between response time and errors, it could be argued that meditators were not performing the task any better, but rather were engaging in a different strategy to the control group (i.e. that they slowed responding in order to make fewer target errors). It is unclear why this effect was only found in the Auditory Target task; however it may be due to the small sample size. Further research should employ larger samples to clarify the speed-accuracy trade-off in regards to crossmodal versions of the SART.

While it was expected that more experienced meditators would have a lower proportion of errors than less experienced meditators, there were no significant correlations found for the visual target- auditory non-target task. There was only one significant correlation found for the auditory target- visual non-target task; a negative correlation between proportion of errors made and frequency of meditation sessions per week. As with the visual and auditory results this is unexpected as there were correlations found with different measures of meditation experience for each task. The lack of a strong association between accuracy at the task and meditation experience is likely due to the measures and sample size of meditators in this study, as other research has supported this association (van Vugt \& Slagter, 2014).

\section{General Discussion}

The present study aimed to find evidence that meditators have superior sustained attention compared to non-meditators. The experiments were designed to also test whether any such advantage was specific to a single modality or was a general effect across modalities. Overall, the results from the three experiments support the hypothesis that meditation brings about positive changes to sustained attentional processing. This effect was found not only for visual sustained attention, but also for auditory sustained attention and a 
crossmodal combination of both visual and auditory attention. Such results are consistent with previous studies that have shown an association between meditation practice and enhanced performance on tasks that measure visual vigilance (Chambers et al., 2008; Maclean et al., 2000), and auditory vigilance (Lutz et al., 2009; Valentine \& Sweet, 1999). Furthermore, the control group results for the visual, auditory and crossmodal experiments mimic a previous study that adopted modality-specific versions of the SART (Seli et al., 2012). This research contributes to the growing number of studies that have found meditation to significantly improve attention, yet this is the first to show that these improvements can be measured through the SART and extend to crossmodal sustained attention.

While this study has shown that participants who regularly engage in meditation have benefits to sustained attention, these results alone cannot infer causation. It cannot be determined whether meditation improves attention, or whether those with better attention are drawn towards meditation. It is also possible that there are third variables that could account for this association. However, this study is supported by experimental research that has shown a causal direction between meditation and attention (Lutz et al., 2009). It is also unlikely that the findings of this study can be explained by individuals with superior attention being drawn towards meditation. Multiple studies have used subjects interested in meditation (in a waitlist for a meditation retreat) as a control group and still found a significant difference between groups (Lutz et al., 2009; Moore et al., 2012). Therefore, the proposal that the practice of meditation positively influences attention is likely. It is also important to note that this study did not involve any active meditation during experimentation and examined the trait rather than state effects. While this study cannot make claims about the state effects of meditation, there is previous research that suggests that being in a state of meditation or immediately following meditation influences attention (Brefczynski-Lewis et al., 2007; Cahn 
\& Polich, 2009). This study does, however, suggest that meditation has long-term, persistent effects on sustained attentional processing.

\section{Modality-Specific versus Supramodal Model of Vigilance}

The present study offers some insight into the debate about whether vigilance is modality-specific or supramodal. The argument for modality-specific vigilance (where visual and auditory vigilance are managed by different mechanisms) can be seen through the differences in visual and auditory tasks. The argument for supramodal model of vigilance (where all modalities are managed by an overarching vigilance system) can be seen through the similarities across tasks when they are equated for difficulty. This will be discussed below.

Modality-specific model of vigilance. The results of this study show there are differences in how individuals perform on the visual and auditory SARTs. These differences support the modality-specific model of vigilance. It was found that there were slower response rates and a lower error rate in the auditory task compared to the visual task (i.e., a reduced vigilance decrement). This difference between the visual and auditory task is consistent with previous studies (Davies \& Parasuraman, 1982; Warm \& Jerison, 1984). There are some possible explanations as to why modality impacts performance in the SART.

The first possible explanation is due to differences in coupling (Hatfield \& Loeb, 1968). It has been proposed that auditory tasks are 'closely coupled' because they usually have some direct link between the stimuli and the individual, such as wearing headphones. Therefore, the orientation of the monitor and the individual has no impact on the receptiveness to the stimuli. In contrast, visual tasks are 'loosely coupled' in that the orientation of the monitor and the individual are crucial in the receptiveness to the stimuli. 
For example, individuals are usually free to make head and eye movements which could reduce the amount of attention focused on the stimuli (Shaw et al., 2009). In order to combat the loose coupling of visual tasks compared to auditory tasks, studies often require participants to sit with their head mounted in a fixed position in front of the monitor. This can lead to discomfort, restlessness and eye strain, which increases workload and reduces the availability of resources for the task (Szalma et al., 2004). While this may explain the differences in performance for some studies, it cannot explain differences for the present study. In the present study participants were required to sit with their heads mounted in a chin rest for the visual, auditory and both crossmodal tasks. Therefore, strain and restlessness would have been fairly consistent across the tasks. Furthermore, a study showed that having eyes open or shut during the auditory SART has no significant change in performance (Seli et al., 2012). We can thus assume that coupling of the individual and the stimuli was consistent across all tasks and did not significantly impact performance in this study.

Another possible explanation is the difference in transduction properties of each modality (Warm \& Jerison, 1984). There is evidence to suggest that while visual stimuli provide more accurate spatial information, auditory stimuli provide more accurate temporal information (Spence, 2007). Furthermore, it may take longer to identify and process the auditory stimuli, in comparison to visual stimuli which are more holistic in their presentation and can be identified quickly (Shen \& Mondor, 2006). Therefore, as individuals take longer to process auditory stimuli, they respond slower which allows for more time to recover from minute lapses of attention. This then reduces the vigilance decrement. This difference in response strategies between the visual and auditory tasks may explain the difference in performance. These differences could suggest that visual and auditory vigilance may be governed by different attentional systems. 
Supramodal model of vigilance. There are clearly modality-specific differences in vigilance tasks. However, these differences can be reduced by equating stimuli discriminability across modalities (Curtindale et al., 2007). This has previously been done by using $d$ '; a signal detection index of perceptual sensitivity (Hatfield \& Soderquist, 1970; Laurie-Rose, Bennett-Murphy, Curtindale, Granger \& Walker, 2005; Loeb \& Binford, 1971). This mathematically derived index allows stimuli discriminability to be set for each individual, therefore matching the difficulty of each task. When each task is equated for difficulty, there are strong correlations between visual, auditory and intermodal vigilance tasks (Tyler, Waag \& Halcomb, 1972). This evidence undermines the argument of a modality-specific model of vigilance.

To further investigate whether vigilance was modality-specific or supramodal, the current study included crossmodal versions of the SART. The results support the argument for a supramodal model. If vigilance was modality-specific, the detection of visual stimuli would not have affected the detection of auditory stimuli and vice versa. However, this was not the case as overall subjects made a large number of errors and switch trials were significantly slower than default trials. This meant that it was effortful for subjects to switch between modalities. This suggests that a single overarching system governs vigilance, as attentional resources are shared across modalities. Future studies should develop further versions of the SART that can measure crossmodal attention across sensory modalities other than visual/auditory. The accumulation of more evidence will give greater support to the argument of the SART being able to measure crossmodal attention and also give support to the argument of a supramodal model of vigilance.

This is further supported by functional magnetic resonance imaging evidence which found similar patterns of activations for visual and auditory attentional networks during a vigilance task (Seidman et al., 1998). A study used transcranial Doppler sonography to 
examine differences in cerebral blood flow between performance on visual and auditory vigilance tasks (Shaw et al., 2009). They equated both tasks for type of stimulus, discrimination difficulty, and display salience. It was found that not only were error rates similar across tasks, but cerebral blood flow velocity (a measure of performance efficiency) declined in a similar manner across the two tasks. This suggests that while there are some important modality-specific features of vigilance, the changes in brain functioning indicative of vigilance performance are independent of sensory modality and support the theory for a supramodal system.

\section{Possible Explanations for Meditation-induced Changes in Attention}

While this study supports the claim that meditation is beneficial to sustained attention, it cannot explain what the mechanisms involved in this process may be. There are, however, some possible explanations as to how meditation may improve attention.

Increases in self-regulation of attention. The most commonly argued mechanism is that the skills and techniques involved in meditation have a direct influence on the selfregulation of attention, and that continuous practice of these techniques can improve attentional processing. As sustained attention has shown to be susceptible to practice effects (Malec, Jones, Rao \& Stubbs, 1984), it is not surprising that these skills may be improved by meditation. This coincides with historical accounts of meditation which outline the processes of improving attention. They describe the practice of learning to stabilise attention on a chosen stimulus, developing the voluntary control of attention, using introspection to determine when attention has wandered and finally guiding attention back to the stimulus (Buddhaghosa, 1979). This repetitive and unwavering practice likely strengthens attentional skills and associated brain regions. 
Improvements in executive control. Relatedly, it is possible that meditation may cause changes in sustained attention through improvements in executive control. Maintaining focus on an intended object (such as breath) while ignoring distractors (such as internal thoughts) engages what is called executive attention or conflict monitoring. Studies have shown that individuals who meditate have superior executive control than non-meditating individuals (Jha et al., 2007; van den Hurk, Giommi, Gielen, Speckens \& Barendregt, 2010). Tang and colleagues (2007) showed that only short periods of meditation practice can improve executive control. These behavioural findings are supported by neuroscientific evidence. The anterior cingulate cortex (ACC) has been established as an area that is widely considered to be involved in the executive control of attention (van Veen \& Carter, 2002). Research has shown that during active meditation, the rostral ACC was highly activated compared to non-meditating individuals (Hölzel et al., 2008). There is also evidence which has shown that meditation practice influences the structure of the ACC. Grant, Courtemanche, Duerden, Duncan and Rainville (2010) showed that cortical thickness of the ACC was greater in experienced meditators than a control group. Another study showed that grey matter volumes in the ACC were increased after a short period of meditation practice (Tang et al., 2010). This research strongly suggests that meditation improves the ability to monitor conflicting stimuli and direct attention accordingly which is accompanied by changes in brain functioning. Therefore, it is likely that improvements in executive control and ACC functioning mediate improvements in sustained attention.

Attentional resource theory. As previously mentioned, the attentional resource theory has been used to describe how the vigilance decrement occurs over time, where there are insufficient attentional resources to adequately complete the task. Perhaps a reduction in the vigilance decrement in meditators could be explained by the attentional resource theory. This is where meditators have either; more attentional resources above baseline, are better 
able to manage and spread their attentional resources, or do not use up so many resources on external factors such as stress or mind wandering. Therefore, meditators would have more resources available to make correct responses throughout the task. It has been proposed by Bishop and colleagues (2004) that meditation increases one's ability to acknowledge extraneous thoughts or sensations and then direct attention back to the point of focus, therefore not elaborating further on these irrelevant perceptions. When attention is released from internal distracters such as thoughts and stress, more attentional resources become available for the task (Bishop et al., 2004). This possibility is further supported by the attentional blink study mentioned previously (Shapiro et al., 1997). In this study individuals had a reduced attentional blink (i.e. they were more frequently able to perceive the second rapidly presented stimulus) after attending a meditation retreat. In addition, the amplitude of the P3b ERP, an index of attentional resources, was significantly reduced for the first target stimulus. This suggests that meditators are better able to allocate and spread attentional resources in order to perceive external stimuli in a balanced and continuous manner (Malinowski, 2013).

Expectancy effects. It could also be proposed that the difference in performance between meditators and the control group was due to expectancy effects. This refers to the process in which a person's expectations may act as a self-fulfilling prophecy (Rosenthal, 1963). This can occur in experimental settings where an experimenter expecting higher ratings from participants gained substantially better scores than experimenters expecting low ratings (Rosenthal \& Fode, 1963). As the experimental group in the current study were recruited from meditation centres and told that they could be involved in research on meditation, there may have been some unavoidable expectations by this group that they were part of research aiming to determine benefits of meditation. Therefore, these individuals may have been more motivated to perform well in the tasks compared to the control individuals. In 
order to avoid this as much as possible neutral language was used when recruiting and no previous effects of meditation were discussed until the completion of the experiments. It is unlikely that meditators were significantly more motivated to perform well as there was no indication of strategic responding. Furthermore, it can be argued that the results of the present study are not due to the control group being abnormally low in arousal and motivation. As the accuracy of the control group has observably similar scores to previous studies that also used the SART, it can be argued that they were a normal sample and did not artificially increase the performance of the meditation group by comparison (Cheyne et al., 2012). In addition, previous studies have controlled for differences in participant motivation, self-selection and experimenter bias between meditators and controls and found no difference in meditation performance (Frumkin, 1979; Yuille \& Sereda, 1980). This makes it unlikely that expectancy effects had a significant influence on the performance between meditators and controls in this study.

Self-related motivations. Another possible explanation as to why practising meditation may influence attention refers to self-related beliefs. It has been shown that the motivation to think favourably of ones future outcomes has an influence on perception. In a study by Balcetis and Dunning (2006), participants were told that they would taste-test drinks and would be randomly allocated to either an orange juice or an unattractive health drink, depending on whether they were presented with a number or a letter. Participants viewed an ambiguous image (B or 13) in a way that would allocate them to the preferred group; the orange juice (Balcetis \& Dunning, 2006). It has been proposed that meditation may reduce these self-related beliefs, thus providing a less cognitively-influenced perception of reality (Hodgins \& Adair, 2010). This claim is relevant as a core aspect of meditation is the gradual reduction of these beliefs (Ghogyam, 1995). Buddhist teachings, which are a foundation for meditation techniques, identify that there is no static, unchanging self as most people would 
assume (Olendzki, 2010). Rather, the perception of 'self' is a series of rapid mental processes that appear as a single entity. With increased meditation practice, individuals report that they can observe these mental processes with clarity (Hölzel et al., 2011). Therefore, individuals who practice meditation regularly are argued to perceive external stimuli with less cognitive driven distortion and less self-related defence (Hodgins, 2008; Hodgins \& Knee, 2002). This may explain how meditation practitioners were able to judge with more accuracy whether the rapid moving stimuli in the SART task were targets or non-targets.

Stress and meditation. Another possible explanation of how meditation improves performance on vigilance tasks is through a reduction in stress. Research has shown that vigilance tasks can induce high levels of stress (Warm, Matthews \& Finomore, 2008). This is supported by studies that have found that epinephrine and norepinephrine levels are increased during vigilance tasks (Frankenhaeuser \& Patkai, 1964; Lundberg \& Frankenhaeuser, 1980). Stress can also impact performance on vigilance tasks. A negative correlation has been between epinephrine levels and performance efficiency (Frankenhaeuser, Nordheden, Myrsten \& Post, 1971; O’Hanlon, 1965). Furthermore, the stress induced by vigilance tasks has been found to be moderated by sensory modality (Szlama et al., 2004). Participants find that visual vigilance tasks are more stressful than auditory vigilance tasks (Galinsky et al., 1993). This is due to the constraints of posture, tension and eye strain during visual compared to auditory tasks. Participants have also shown to recover much faster from stress after auditory tasks (Szlama et al., 2004). This may explain in part why the vigilance decrement was reduced in the auditory compared to the visual task.

Individuals who meditate may be less impacted by the effects of stress during vigilance tasks. There are numerous studies that have shown a decrease in stress in normal populations after meditation (Astin, 1997; Oman, Shapiro, Thoresen, Plante \& Flinders, 2008; Shapiro, Schwartz \& Bonner, 1998). For example, Tang and colleagues (2007) showed 
that after five days of IBMT individuals showed signs of reduced stress. They had reduced levels of cortisol and more immunoreactivity than a relaxation control group. This suggests that meditators have benefits in their ability to cope with stress. Therefore, this may be a contributing factor in the superior attentional processing of meditators.

Together, the explanations outlined above provide possible mechanisms that may be involved in the association between meditation and improvements in sustained attention. Future research should further examine these factors to determine their role in the association between meditation and attention.

\section{Validity of the SART}

While the Sustained Attention to Response task has been argued as a more sensitive measure of sustained attention than traditional vigilance tasks, it has also been criticised for its limitations in regards to a speed-accuracy trade-off and whether it really measures sustained attention. These criticisms are addressed below.

Speed-accuracy trade-off. A frequently mentioned criticism of the SART is the occurrence of a speed-accuracy trade-off. This is where participants can rely on strategies to either reduce response speed in order to perform more accurately, or to increase response speed while sacrificing accuracy (Helton, Kern \& Walker, 2009). No evidence of a speedaccuracy trade-off was found in the current study, with the exception of the Auditory Target Experiment. Overall, this shows that the advantage of accuracy that meditators had was not artificially inflated by their slower responding. It is unclear exactly why the control group responded significantly faster for the auditory and crossmodal tasks, and why there was a correlation for meditators between response time and error only for the Auditory Target Experiment. Perhaps the meditation group had a greater expectancy to perform well and so interpreted the instructions with higher emphasis on accuracy over speed. While the 
commonly given instructions are to give equal importance to both speed and accuracy, it is important to note that there can be individual differences in the interpretation of these instructions (Howell \& Kreidler, 1963; Imbo \& Vandierendonck, 2010). Even though the occurrence of a speed-accuracy trade-off was found in the Auditory Target Experiment, this did not occur in the other crossmodal experiment. Therefore, it is likely that this effect was due to experimental limitations.

It is argued by Robertson and colleagues (1997) that the speed-accuracy trade-off is an essential component of the SART. Lapses of attention are expressed through automatic responding, which in essence are rapid, leading to an increase in errors. However, others argue that the rapid responding may not indicate lapses of attention. Rather, the stimuli could be processed and yet the automatic motor response not inhibited; leading to an error (Seli et al., 2012). While this issue makes the interpretation of errors difficult, there is some electrophysiological evidence to suggest that errors are mostly associated with lapses of attention. A study by Manly and colleagues (2000) showed that a reduction in the P300, an ERP component commonly related to attention, was predictive of subsequent errors. More importantly, this effect was independent of response time. Another study showed that the SART measures sustained attention regardless of the speed-accuracy trade-off (Manly et al., 2000). They eliminated the variability in RT by requiring participants to respond on cue. They were then able to determine whether the errors made would reduce (therefore supporting a response inhibition explanation) or stay consistent (supporting an attentional lapse explanation). They found that despite a successful manipulation of RTs, the error rates did not reduce, suggesting that attentional control was the most important predictor of accuracy in the SART.

Response inhibition. The SART has also been criticised as measuring response inhibition rather than sustained attention (Stevenson, Russell \& Helton, 2011). This is 
because subjects may be perceptually aware of the target stimuli, but unable to withhold the automatic non-target motor response, therefore making an error. One study compared two vigilance response formats; the traditional format where participants only respond to target stimuli, and the SART format where participants only withhold responses for target stimuli (Helton, 2009). There was a significant difference found in error rates between the two response formats, in that the SART task yielded more errors, even though identical stimuli were used in both tasks. It was suggested that the higher error rates in the SART were due to failures of response inhibition rather than a failure to detect the target due to an attention lapse. Therefore, the SART is likely a compound measure of sustained attention and response inhibition.

However, Robertson and colleagues (1997) had previously asserted that the SART was significantly correlated with measures of sustained attention and not with other measures of response inhibition. While the present study cannot directly address these criticisms, it can be argued that given the converging evidence from the current study combined with the previous research, it is likely that meditation affects attention rather than response inhibition. Moreover, it is important to note that the above criticisms only refer to the original Robertson and colleagues (1997) SART and do not extend to Cheyne and colleagues' (2012) version where a response switch is required instead of withholding a response for target stimuli - the version used in the current study.

\section{Implications}

Meditation has long been used to focus attention, improve general well-being and heighten spiritual awareness; yet until recently meditation was an unfamiliar concept for the Western world. By examining the behavioural benefits and associated neuropsychological changes through scientific approach it could open up the process of meditation to a far 
reaching audience who would have otherwise not considered this technique. It will not only further optimise the use of meditation based therapies for treatment of mental and physical conditions, but also better inform the general population who wish to incorporate meditation into their daily lives in order to reap its benefits (Ainsworth et al., 2013).

Deficits in sustained attention are major symptoms of disorders such as Schizophrenia (Levin, Wilson, Rose \& McEvoy, 1996), Attention Deficit Hyperactivity disorder (Seidel \& Joschko, 1990), Autism Spectrum disorder (Garretson, Fein \& Waterhouse, 1990), bipolar disorder (Clark, Iversen \& Goodwin, 2002) and Alzheimer's disease (Berardi, Parasuraman \& Haxby, 2005). Determining how individuals who regularly meditate differ in their brain function and structure to non-meditating counterparts could assist in the development of psychiatric models and developed aetiologies for these disorders (Shaw et al., 2009). It could also help develop therapies and treatment programs that incorporate meditation. There are currently already meditation based treatments such as the Mindfulness Based Stress Reduction and Mindfulness Based Cognitive Therapy (Kabat-Zinn, 2003a; Kabat-Zinn, 2003b). So far these therapies have shown to have beneficial results in the treatment of pain, stress, anxiety, depression and eating disorders (Baer, 2003).

In addition to treatments for psychopathologies, there is a market for methods to improve attention in healthy populations. There are many jobs that rely on individuals' abilities to sustain attention for long periods of time (Barger et al., 2006). The decline in sustained attention could cause injury or be fatal. Many workplaces could utilise meditation techniques in order to maintain a high level of competency across these attention demanding roles, therefore reducing risk and injury. 


\section{Concluding Remarks}

The current research aimed to investigate whether meditation has a positive influence on the ability to sustain attention. Of particular interest was whether these benefits would extend across sensory modalities. This is the first study to show that individuals who regularly meditate have superior performance on the Sustained Attention to Response Task compared to non-meditating counterparts. This was true for visual, auditory and the visualtarget crossmodal version of the SART. This research contributes to the growing number of studies that have found a positive association between meditation and superior attentional, perceptual and cognitive abilities. This research also gives support to the use of the SART as a measure of sustained attention and provides an example of how it could be used to measure vigilance across different modalities. The implications of such research could assist not only pathological populations with non-medicinal treatment options, but could also impact the general population within the workplace where sustained attention is becoming increasingly more relevant. 


\section{References}

Ainsworth, B., Eddershaw, R., Meron, D., Baldwin, D. S., \& Garner, M. (2013). The effect of focused attention and open monitoring meditation on attention network function in healthy volunteers. Psychiatry research, 210(3), 1226-1231.

Astin, J. A. (1997). Stress reduction through mindfulness meditation. Psychotherapy and psychosomatics, 66(2), 97-106.

Baer, R. A. (2003). Mindfulness training as a clinical intervention: A conceptual and empirical review. Clinical psychology: Science and practice, 10(2), 125-143.

Balcetis, E., \& Dunning, D. (2006). See what you want to see: motivational influences on visual perception. Journal of personality and social psychology, 91(4), 612.

Barger, L. K., Ayas, N. T., Cade, B. E., Cronin, J. W., Rosner, B., Speizer, F. E., \& Czeisler, C. A. (2006). Impact of extended-duration shifts on medical errors, adverse events, and attentional failures. PLoS medicine, 3(12), 487.

Barkley, R. A., \& Cox, D. (2007). A review of driving risks and impairments associated with attention-deficit/hyperactivity disorder and the effects of stimulant medication on driving performance. Journal of safety research, 38(1), 113-128.

Berardi, A. M., Parasuraman, R., \& Haxby, J. V. (2005). Sustained attention in mild Alzheimer's disease. Developmental neuropsychology, 28(1), 507-537.

Binford, J. R., \& Loeb, M. (1966). Changes within and over repeated sessions in criterion and effective sensitivity in an auditory vigilance task. Journal of Experimental Psychology, 72(3), 339. 
Bishop, S. R., Lau, M., Shapiro, S., Carlson, L., Anderson, N. D., Carmody, J., \& Devins, G. (2004). Mindfulness: A proposed operational definition. Clinical psychology: Science and practice, 11(3), 230-241.

Boorstein, S. (1996). Clinical aspects of meditation. Textbook of transpersonal psychiatry and psychology, 344-354.

Brefczynski-Lewis, J. A., Lutz, A., Schaefer, H. S., Levinson, D. B., \& Davidson, R. J. (2007). Neural correlates of attentional expertise in long-term meditation practitioners. Proceedings of the national Academy of Sciences, 104(27), 1148311488.

Breitmeyer, B., \& Öğmen, H. (2006). Visual masking: Time slices through conscious and unconscious vision (41). Oxford University Press.

Brown, D., Forte, M., \& Dysart, M. (1984). Visual sensitivity and mindfulness meditation. Perceptual and motor skills, 58(3), 775-784.

Buddhaghosa. (1979). The path of purification (Nanamoli B. Trans.). Sri Lanka: Buddhist Publication Society.

Cahn, B. R., Delorme, A., \& Polich, J. (2012). Event-related delta, theta, alpha, and gamma correlates to auditory oddball processing during Vipassana meditation. Social cognitive and affective neuroscience, 60.

Cahn, B. R., \& Polich, J. (2009). Meditation (Vipassana) and the P3a event-related brain potential. International Journal of Psychophysiology, 72(1), 51-60.

Cardoso, R., De Souza, E., Camano, L., \& Leite, J. R. (2007). Prefrontal Cortex in Meditation. NeuroQuantology, 5(2), 233-240. 
Carr, G. D., \& White, N. M. (1986). Anatomical disassociation of amphetamine's rewarding and aversive effects: an intracranial microinjection study. Psychopharmacology, 89(3), 340-346.

Chambers, R., Lo, B. C. Y., \& Allen, N. B. (2008). The impact of intensive mindfulness training on attentional control, cognitive style, and affect. Cognitive Therapy and Research, 32(3), 303-322.

Cheyne, J. A., Carriere, J. S., \& Smilek, D. (2006). Absent-mindedness: Lapses of conscious awareness and everyday cognitive failures. Consciousness and cognition, 15(3), 578592.

Cheyne, D. O., Ferrari, P., \& Cheyne, J. A. (2012). Intended actions and unexpected outcomes: automatic and controlled processing in a rapid motor task. Frontiers in human neuroscience, 6 .

Cheyne, J. A., Solman, G. J., Carriere, J. S., \& Smilek, D. (2009). Anatomy of an error: A bidirectional state model of task engagement/disengagement and attention-related errors. Cognition, 111(1), 98-113.

Chun, M. M., \& Potter, M. C. (1995). A two-stage model for multiple target detection in rapid serial visual presentation. Journal of Experimental psychology: Human perception and performance, 21(1), 109.

Clark, L., Iversen, S. D., \& Goodwin, G. M. (2002). Sustained attention deficit in bipolar disorder. The British Journal of Psychiatry, 180(4), 313-319.

Cohen, J. (1988). Statistical power analysis: A computer program. Routledge. 
Cohen, R. M., Semple, W. E., Gross, M., King, A. C., \& Nordahl, T. E. (1992). Metabolic brain pattern of sustained auditory discrimination. Experimental Brain Research, 92(1), 165-172.

Curtindale, L., Laurie-Rose, C., Bennett-Murphy, L., \& Hull, S. (2007). Sensory modality, temperament, and the development of sustained attention: A vigilance study in children and adults. Developmental psychology, 43(3), 576.

Davies, D. R., \& Parasuraman, R. (1982). The psychology of vigilance. Academic Press.

Delgado-Pastor, L. C., Perakakis, P., Subramanya, P., Telles, S., \& Vila, J. (2013). Mindfulness (Vipassana) meditation: Effects on P3b event-related potential and heart rate variability. International Journal of Psychophysiology, 90(2), 207-214.

Di Nardo, P. A., \& Raymond, J. B. (1979). Locus of control and attention during meditation. Journal of Consulting and Clinical Psychology, 47(6), 1136.

Driver, J., \& Spence, C. (1998). Attention and the crossmodal construction of space. Trends in cognitive sciences, 2(7), 254-262.

Frankenhaeuser, M., Nordheden, B., Myrsten, A. L., \& Post, B. (1971). Psychophysiological reactions to understimulation and overstimulation. Acta Psychologica, 35(4), 298-308.

Frankenhaeuser, M., \& Pátkai, P. (1964). Catecholamine excretion and performance during stress. Perceptual and motor skills, 19, 13.

Frankmann, J. P., \& Adams, J. (1962). Theories of vigilance. Psychological Bulletin, 59(4), 257.

Frumkin, L. R., \& Pagano, R. R. (1979). The effect of transcendental meditation on iconic memory. Biofeedback and self-regulation, 4(4), 313-322. 
Galinsky, T. L., Rosa, R. R., Warm, J. S., \& Dember, W. N. (1993). Psychophysical determinants of stress in sustained attention. Human Factors: The Journal of the Human Factors and Ergonomics Society, 35(4), 603-614.

Garretson, H. B., Fein, D., \& Waterhouse, L. (1990). Sustained attention in children with autism. Journal of autism and developmental disorders, 20(1), 101-114.

Ghogyam, T. (1995). The path is the goal. Boston-London 1995.

Goldstone, S., \& Goldfarb, J. L. (1966). The perception of time by children. Perceptual development in children, 445-486.

Grant, J. A., Courtemanche, J., Duerden, E. G., Duncan, G. H., \& Rainville, P. (2010). Cortical thickness and pain sensitivity in zen meditators. Emotion,10(1), 43.

Gruber, A. (1964). Sensory alternation and performance in a vigilance task. Human Factors: The Journal of the Human Factors and Ergonomics Society,6(1), 3-12.

Gunaratana, H. (1991). Mindfulness in plain English. Shambhala, Boston.

Hanslmayr, S., Aslan, A., Staudigl, T., Klimesch, W., Herrmann, C. S., \& Bäuml, K. H. (2007). Prestimulus oscillations predict visual perception performance between and within subjects. Neuroimage, 37(4), 1465-1473.

Hatfield, J. L., \& Loeb, M. (1968). Sense mode and coupling in a vigilance task. Perception \& Psychophysics, 4(1), 29-36.

Hatfield, J. L., \& Soderquist, D. R. (1970). Coupling Effects and Performance in Vigilance Tasks. Human Factors: The Journal of the Human Factors and Ergonomics Society, 12(4), 351-359. 
Helton, W. S. (2009). Impulsive responding and the sustained attention to response task. Journal of Clinical and Experimental Neuropsychology, 31(1), 39-47.

Helton, W. S., Kern, R. P., \& Walker, D. R. (2009). Conscious thought and the sustained attention to response task. Consciousness and cognition, 18(3), 600-607.

Hitchcock, E. M., Warm, J. S., Matthews, G., Dember, W. N., Shear, P. K., Tripp, L. D., \& Parasuraman, R. (2003). Automation cueing modulates cerebral blood flow and vigilance in a simulated air traffic control task. Theoretical Issues in Ergonomics Science, 4(1-2), 89-112.

Hodgins, H. S., \& Adair, K. C. (2010). Attentional processes and meditation. Consciousness and cognition, 19(4), 872-878.

Hodgins, H. S. (2008). Motivation, threshold for threat, and quieting the ego. In Wayment H. \& Bauer J. (Eds.), Transcending self-interest: Psychological explorations of the quiet ego (pp. 117-124). Washington, DC: American Psychological Association.

Hodgins, H. S., \& Knee, C. R. (2002). The integrating self and conscious experience. Handbook of self-determination research, 87-100.

Hölzel, B. K., Lazar, S. W., Gard, T., Schuman-Olivier, Z., Vago, D. R., \& Ott, U. (2011). How does mindfulness meditation work? Proposing mechanisms of action from a conceptual and neural perspective. Perspectives on Psychological Science, 6(6), 537559.

Hölzel, B. K., Ott, U., Gard, T., Hempel, H., Weygandt, M., Morgen, K., \& Vaitl, D. (2008). Investigation of mindfulness meditation practitioners with voxel-based morphometry. Social cognitive and affective neuroscience, 3(1), 55-61. 
Howell, W. C., \& Kreidler, D. L. (1963). Information processing under contradictory instructional sets. Journal of Experimental Psychology, 65(1), 39.

Imbo, I., \& Vandierendonck, A. (2010). Instruction and load effects on high-skill and lowskill individuals: A study in the domain of mental arithmetic. European Journal of Cognitive Psychology, 22(6), 964-989.

Jensen, O., \& Lisman, J. E. (1996). Hippocampal CA3 region predicts memory sequences: accounting for the phase precession of place cells. Learning \& Memory, 3(2-3), 279287.

Jha, A. P., Krompinger, J., \& Baime, M. J. (2007). Mindfulness training modifies subsystems of attention. Cognitive, Affective, \& Behavioral Neuroscience, 7(2), 109-119.

Josipovic, Z. (2010). Duality and nonduality in meditation research. Consciousness and cognition, 19(4), 1119-1121.

Kabat-Zinn, J. (1982). An outpatient program in behavioral medicine for chronic pain patients based on the practice of mindfulness meditation: Theoretical considerations and preliminary results. General hospital psychiatry, 4(1), 33-47.

Kabat-Zinn, J. (2003a). Mindfulness-based interventions in context: past, present, and future. Clinical psychology: Science and practice, 10(2), 144-156.

Kabat-Zinn, J. (2003b). Mindfulness-based stress reduction (MBSR).Constructivism in the Human Sciences.

Kahana, M. J., Seelig, D., \& Madsen, J. R. (2001). Theta returns. Current opinion in neurobiology, 11(6), 739-744.

Kahneman, D. (1973). Attention and effort (p. 246). Englewood Cliffs: Prentice-Hall. 
Kapleau, R. P. (2013). The three pillars of Zen. Anchor Press.

Kasamatsu, A., \& Hirai, T. (1966). An electroencephalographic study on the Zen meditation (Zazen). Psychiatry and Clinical Neurosciences, 20(4), 315-336.

Kerns, K. A., Eso, K., \& Thomson, J. (1999). Investigation of a direct intervention for improving attention in young children with ADHD. Developmental neuropsychology, 16(2), 273-295.

Klimesch, W., Schack, B., Schabus, M., Doppelmayr, M., Gruber, W., \& Sauseng, P. (2004). Phase-locked alpha and theta oscillations generate the P1-N1 complex and are related to memory performance. Cognitive Brain Research, 19(3), 302-316.

Knowles, D., \& Tay, R. S. (2002). Driver inattention: more risky than the fatal four?. Transportation Research Part F: Traffic Psychology and Behaviour.

Koelega, H. S. (1993). Stimulant drugs and vigilance performance: a review. Psychopharmacology, 111(1), 1-16.

Kristeller, J. L., \& Hallett, C. B. (1999). An exploratory study of a meditation-based intervention for binge eating disorder. Journal of Health Psychology, 4(3), 357-363.

Kuyken, W., Byford, S., Taylor, R. S., Watkins, E., Holden, E., White, K., \& Teasdale, J. D. (2008). Mindfulness-based cognitive therapy to prevent relapse in recurrent depression. Journal of consulting and clinical psychology, 76(6), 966.

Laurie-Rose, C., Bennett-Murphy, L., Curtnindale, L. M., Granger, A. L., \& Walker, H. B. (2005). Equating tasks and sustaining attention in children and adults: The methodological and theoretical utility ofd'matching. Perception \& psychophysics, 67(2), 254-263. 
Lazar, S. W., Kerr, C. E., Wasserman, R. H., Gray, J. R., Greve, D. N., Treadway, M. T., \& Fischl, B. (2005). Meditation experience is associated with increased cortical thickness. Neuroreport, 16(17), 1893.

Levin, E. D., Wilson, W., Rose, J. E., \& McEvoy, J. (1996). Nicotine-haloperidol interactions and cognitive performance in schizophrenics. Neuropsychopharmacology, 15(5), 429436.

Loeb, M., \& Binford, J. R. (1968). Variation in performance on auditory and visual monitoring tasks as a function of signal and stimulus frequencies. Perception \& Psychophysics, 4(6), 361-367.

Luders, E., Kurth, F., Mayer, E. A., Toga, A. W., Narr, K. L., \& Gaser, C. (2012). The unique brain anatomy of meditation practitioners: alterations in cortical gyrification. Frontiers in human neuroscience, 6.

Lundberg, U., \& Frankenhaeuser, M. (1980). Pituitary-adrenal and sympathetic-adrenal correlates of distress and effort. Journal of Psychosomatic Research, 24(3), 125-130.

Lutz, A., Slagter, H. A., Dunne, J. D., \& Davidson, R. J. (2008). Attention regulation and monitoring in meditation. Trends in cognitive sciences, 12(4), 163-169.

Lutz, A., Slagter, H. A., Rawlings, N. B., Francis, A. D., Greischar, L. L., \& Davidson, R. J. (2009). Mental training enhances attentional stability: neural and behavioral evidence. The Journal of Neuroscience, 29(42), 13418-13427.

MacLean, K. A., Ferrer, E., Aichele, S. R., Bridwell, D. A., Zanesco, A. P., Jacobs, T. L., \& Saron, C. D. (2010). Intensive meditation training improves perceptual discrimination and sustained attention. Psychological science,21(6), 829-839. 
Malec, J., Jones, R., Rao, N., \& Stubbs, K. (1984). Video game practice effects on sustained attention in patients with craniocerebral trauma. Cognitive Rehabilitation.

Malinowski, P. (2013). Neural mechanisms of attentional control in mindfulness meditation. Frontiers in neuroscience, 7.

Manly, T., Davison, B., Heutink, J., Galloway, M., \& Robertson, I. H. (2000). Not enough time or not enough attention? Speed, error and self-maintained control in the Sustained Attention to Response Test (SART). Clinical Neuropsychological Assessment, 3, 167-177.

Marchand, W. R. (2012). Mindfulness-based stress reduction, mindfulness-based cognitive therapy, and Zen meditation for depression, anxiety, pain, and psychological distress. Journal of Psychiatric Practice ${ }^{\circledR}, 18(4), 233-252$.

Martin, M. (1978). Retention of attended and unattended auditorily and visually presented material. The Quarterly Journal of Experimental Psychology, 30(2), 187-200.

Martin, M. (1983). Cognitive failure: Everyday and laboratory performance. Bulletin of the Psychonomic Society, 21(2), 97-100.

Monk-Turner, E. (2003). The benefits of meditation: experimental findings. The Social Science Journal, 40(3), 465-470.

Moore, A., Gruber, T., Derose, J., \& Malinowski, P. (2012). Regular, brief mindfulness meditation practice improves electrophysiological markers of attentional control. Frontiers in human neuroscience, 6.

Neal, G. L., \& Pearson, R. G. (1966). Comparative effects of age, sex, and drugs upon two tasks of auditory vigilance. Perceptual and motor skills, 23(3), 967-974. 
Niedermeyer, E. (1997). Alpha rhythms as physiological and abnormal phenomena. International Journal of Psychophysiology, 26(1-3), 31-49.

O'Hanlon, J. F. (1965). Adrenaline and noradrenaline: Relation to performance in a visual vigilance task. Science, 150(3695), 507-509.

Olendzki, A. (2010). Unlimiting mind: The radically experiential psychology of Buddhism. Simon and Schuster.

Oman, D., Shapiro, S. L., Thoresen, C. E., Plante, T. G., \& Flinders, T. (2008). Meditation lowers stress and supports forgiveness among college students: A randomized controlled trial. Journal of American College Health, 56(5), 569-578.

Parasuraman, R., \& Giambra, L. (1991). Skill development in vigilance: effects of event rate and age. Psychology and aging, 6(2), 155.

Parasuraman, R., Mutter, S. A., \& Molloy, R. (1991). Sustained attention following mild closed-head injury. Journal of Clinical and Experimental Neuropsychology, 13(5), 789-811.

Pardo, J. V., Fox, P. T., \& Raichle, M. E. (1991). Localization of a human system for sustained attention by positron emission tomography. Nature, 349(6304), 61-64.

Peirce, J. W. (2007). PsychoPy—psychophysics software in Python. Journal of neuroscience methods, 162(1), 8-13.

Posner, M. I. (1980). Orienting of attention. Quarterly journal of experimental psychology, 32(1), 3-25.

Potter, M. C., Chun, M. M., Banks, B. S., \& Muckenhoupt, M. (1998). Two attentional deficits in serial target search: the visual attentional blink and an amodal task-switch 
deficit. Journal of Experimental Psychology: Learning, Memory, and Cognition, 24(4), 979.

Rabbitt, P., \& Abson, V. (1990). 'Lost and Found': Some logical and methodological limitations of self-report uestionnaires as tools to study cognitive ageing. British Journal of Psychology, 81(1), 1-16.

Rao, S. M., Leo, G. J., Haughton, V. M., Aubin-Faubert, P. S., \& Bernardin, L. (1989). Correlation of magnetic resonance imaging with neuropsychological testing in multiple sclerosis. Neurology, 39(2), 161-161.

Rensink, R. A., O'Regan, J. K., \& Clark, J. J. (1997). To see or not to see: The need for attention to perceive changes in scenes. Psychological science, 8(5), 368-373.

Repp, B. H., \& Penel, A. (2002). Auditory dominance in temporal processing: new evidence from synchronization with simultaneous visual and auditory sequences. Journal of Experimental Psychology: Human Perception and Performance, 28(5), 1085.

Rizzi, F. (Ed.). (2005). The Way of Awakening: A Commentary on Shantideva's Bodhicharyavatara. Wisdom Publications Inc.

Robertson, I. H., Manly, T., Andrade, J., Baddeley, B. T., \& Yiend, J. (1997). Oops!': performance correlates of everyday attentional failures in traumatic brain injured and normal subjects. Neuropsychologia, 35(6), 747-758.

Rosenthal, R. (1963). On the social psychology of the psychological experiment: 1, 2 the experimenter's hypothesis as unintended determinant of experimental results. American Scientist, 268-283. 
Rosenthal, R., \& Fode, K. L. (1963). The effect of experimenter bias on the performance of the albino rat. Behavioral Science, 8(3), 183-189.

Rueckert, L., Sorensen, L., \& Levy, J. (1994). Callosal efficiency is related to sustained attention. Neuropsychologia, 32(2), 159-173.

Sarter, M., Givens, B., \& Bruno, J. P. (2001). The cognitive neuroscience of sustained attention: where top-down meets bottom-up. Brain research reviews,35 (2), 146-160.

Schmertz, S. K., Anderson, P. L., \& Robins, D. L. (2009). The relation between self-report mindfulness and performance on tasks of sustained attention. Journal of Psychopathology and Behavioral Assessment, 31(1), 60-66.

Segal, Z. V., Williams, J. M. G., \& Teasdale, J. D. (2002). Mindfulness-based cognitive therapy for depression: A new approach to relapse prevention.

Seidel, W. T., \& Joschko, M. (1990). Evidence of difficulties in sustained attention in children with ADDH. Journal of abnormal child psychology, 18(2), 217-229.

Seidman, L. J., Breiter, H. C., Goodman, J. M., Goldstein, J. M., Woodruff, P. W., O'Craven, K., \& Rosen, B. R. (1998). A functional magnetic resonance imaging study of auditory vigilance with low and high information processing demands. Neuropsychology, 12(4), 505.

Seli, P., Cheyne, J. A., Barton, K. R., \& Smilek, D. (2012). Consistency of sustained attention across modalities: Comparing visual and auditory versions of the SART. Canadian Journal of Experimental Psychology/Revue canadienne de psychologie expérimentale, 66(1), 44. 
Semrud-Clikeman, M., Steingard, R. J., Filipek, P., Biederman, J., Bekken, K., \& Renshaw, P. F. (2000). Using MRI to examine brain-behavior relationships in males with attention deficit disorder with hyperactivity. Journal of the American Academy of Child \& Adolescent Psychiatry, 39(4), 477-484.

Shapiro, K. L., Raymond, J. E., \& Arnell, K. M. (1997). The attentional blink.Trends in cognitive sciences, 1(8), 291-296.

Shapiro, S. L., Schwartz, G. E., \& Bonner, G. (1998). Effects of mindfulness-based stress reduction on medical and premedical students. Journal of behavioral medicine, 21(6), 581-599.

Shapiro, D. H., \& Walsh, R. N. (Eds.). (1984). Meditation: Classic and contemporary perspectives. Aldine Transaction.

Shaw, T. H., Warm, J. S., Finomore, V., Tripp, L., Matthews, G., Weiler, E., \& Parasuraman, R. (2009). Effects of sensory modality on cerebral blood flow velocity during vigilance. Neuroscience letters, 461(3), 207-211.

Shen, D., \& Mondor, T. A. (2006). Effect of distractor sounds on the auditory attentional blink. Perception \& psychophysics, 68(2), 228-243.

Sheridan, T. B. (1980). Computer control and human alienation. Technology review, 83(1), $65-73$.

Simons, D. J., \& Chabris, C. F. (1999). Gorillas in our midst: Sustained inattentional blindness for dynamic events. Perception-London, 28(9), 1059-1074. 
Slagter, H. A., Lutz, A., Greischar, L. L., Francis, A. D., Nieuwenhuis, S., Davis, J. M., \& Davidson, R. J. (2007). Mental training affects distribution of limited brain resources. PLoS biology, 5(6), 138.

Smilek, D., Carriere, J. S., \& Cheyne, J. A. (2010). Out of mind, out of sight eye blinking as indicator and embodiment of mind wandering. Psychological Science, 21(6), 786-789.

Speca, M., Carlson, L. E., Goodey, E., \& Angen, M. (2000). A randomized, wait-list controlled clinical trial: the effect of a mindfulness meditation-based stress reduction program on mood and symptoms of stress in cancer outpatients. Psychosomatic medicine, 62(5), 613-622.

Spence, C. (2007). Audiovisual multisensory integration. Acoustical science and technology, 28(2), 61-70.

Stevenson, H., Russell, P. N., \& Helton, W. S. (2011). Search asymmetry, sustained attention, and response inhibition. Brain and cognition, 77(2), 215-222.

Stroop, J. R. (1935). Studies of interference in serial verbal reactions. Journal of experimental psychology, 18(6), 643.

Sturm, W., Willmes, K., Orgass, B., \& Hartje, W. (1997). Do specific attention deficits need specific training?. Neuropsychological Rehabilitation, 7(2), 81-103.

Szalma, J. L., Warm, J. S., Matthews, G., Dember, W. N., Weiler, E. M., Meier, A., \& Eggemeier, F. T. (2004). Effects of sensory modality and task duration on performance, workload, and stress in sustained attention. Human Factors: The Journal of the Human Factors and Ergonomics Society, 46(2), 219-233. 
Takahashi, M., Iwamoto, K., Fukatsu, H., Naganawa, S., Iidaka, T., \& Ozaki, N. (2010). White matter microstructure of the cingulum and cerebellar peduncle is related to sustained attention and working memory: a diffusion tensor imaging study. Neuroscience letters, 477(2), 72-76.

Tang, Y. Y., Lu, Q., Geng, X., Stein, E. A., Yang, Y., \& Posner, M. I. (2010). Short-term meditation induces white matter changes in the anterior cingulate. Proceedings of the National Academy of Sciences, 107(35), 15649-15652.

Tang, Y. Y., Ma, Y., Fan, Y., Feng, H., Wang, J., Feng, S., \& Fan, M. (2009). Central and autonomic nervous system interaction is altered by short-term meditation. Proceedings of the national Academy of Sciences, 106(22), 8865-8870.

Tang, Y. Y., Ma, Y., Wang, J., Fan, Y., Feng, S., Lu, Q., \& Posner, M. I. (2007). Short-term meditation training improves attention and self-regulation. Proceedings of the National Academy of Sciences, 104(43), 17152-17156.

Temple, J. G., Warm, J. S., Dember, W. N., Jones, K. S., LaGrange, C. M., \& Matthews, G. (2000). The effects of signal salience and caffeine on performance, workload, and stress in an abbreviated vigilance task. Human Factors: The Journal of the Human Factors and Ergonomics Society, 42(2), 183-194.

Tyler, D. M., Waag, W. L., \& Halcomb, C. G. (1972). Monitoring performance across sense modes: an individual differences approach. Human Factors: The Journal of the Human Factors and Ergonomics Society, 14(6), 539-547.

Valentine, E. R. (1988). Does meditation affect attention. SIGMA Newsletter, 4, 15-17. 
Valentine, E. R., \& Sweet, P. L. (1999). Meditation and attention: A comparison of the effects of concentrative and mindfulness meditation on sustained attention. Mental Health, Religion \& Culture, 2(1), 59-70.

van den Hurk, P. A., Giommi, F., Gielen, S. C., Speckens, A. E., \& Barendregt, H. P. (2010). Greater efficiency in attentional processing related to mindfulness meditation. The Quarterly Journal of Experimental Psychology, 63(6), 1168-1180.

Van Nuys, D. (1971). A novel technique for studying attention during meditation. Journal of Transpersonal Psychology.

Van Veen, V., \& Carter, C. S. (2002). The timing of action-monitoring processes in the anterior cingulate cortex. Journal of cognitive neuroscience,14(4), 593-602.

van Vugt, M. K., \& Slagter, H. A. (2014). Control over experience? Magnitude of the attentional blink depends on meditative state. Consciousness and cognition, 23, 32-39.

Verster, J. C., \& Roth, T. (2013). Vigilance decrement during the on-the-road driving tests: The importance of time-on-task in psychopharmacological research. Accident Analysis \& Prevention, 58, 244-248.

Wallace, B. A. (2005). Balancing the mind: A Tibetan Buddhist approach to refining attention. Snow Lion Publications.

Warm, J. S. (1993). Vigilance and target detection. Workload transition: Implications for individual and team performance, 2000.

Warm, J. S., Dember, W. N., \& Hancock, P. A. (1996). Vigilance and workload in automated systems. 
Warm, J. S., \& Jerison, H. J. (1984). The psychophysics of vigilance. In J. S. Warm (Ed.), Sustained attention in human performance (pp. 15-59). UK: Wiley.

Warm, J. S., Matthews, G., \& Finomore Jr, V. S. (2008). Vigilance, workload, and stress. Performance under stress, 115-41.

Warm, J. S., Parasuraman, R., \& Matthews, G. (2008). Vigilance requires hard mental work and is stressful. Human Factors: The Journal of the Human Factors and Ergonomics Society, 50(3), 433-441.

Wickens, C. D. (1981). Processing Resources in Attention, Dual Task Performance, and Workload Assessment. University of Illinois: Engineering-psychology research laboratory.

Wiener, E. L. (1968). Training for vigilance: Repeated sessions with knowledge of results. Ergonomics, 11(6), 547-556.

Wilkins, A. J., Shallice, T., \& McCarthy, R. (1987). Frontal lesions and sustained attention. Neuropsychologia, 25(2), 359-365.

Wyble, B., Bowman, H., \& Nieuwenstein, M. (2009). The attentional blink provides episodic distinctiveness: sparing at a cost. Journal of Experimental Psychology: Human Perception and Performance, 35(3), 787.

Yuille, J. C., \& Sereda, L. (1980). Positive effects of meditation: A limited generalization?. Journal of Applied Psychology, 65(3), 333. 


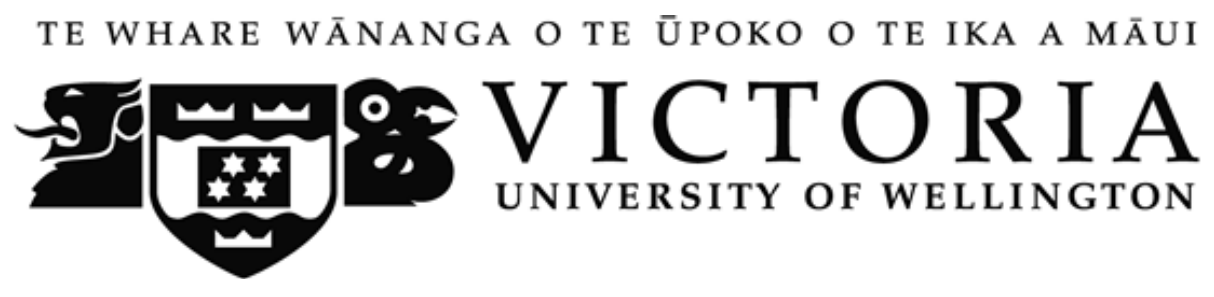

Visual and auditory attentional processing and meditation

Paige Badart (MSc Student) paige.badart@vuw.ac.nz

Dr Steve Prime (Lecturer)

Dr John McDowall (Senior Lecturer) john.mcdowall@vuw.ac.nz (04) 463-6423

What is the purpose of this research?

- This study is investigating how the ability to focus attention may be different for individuals who practice meditation compared to non-meditators.

Who is conducting the research?

- We are a team of researchers in the Schools of Psychology at Victoria University of Wellington. Paige Badart is a Masters student and Drs John McDowall and Steve Prime are researchers in the School of Psychology at Victoria University of Wellington. This research has been approved by the School of Psychology Human Ethics Committee under delegated authority of Victoria University of Wellington's Human Ethics Committee.

\section{What is involved if you agree to participate?}

- If you agree to participate in this study, you may be asked questions about your experience with meditation. We will then perform a simple task on the computer. You will be asked to press a button every time a repetitive visual stimulus appears on the computer screen or a repetitive sound is played over the headphones. You will be asked to withhold your response when you see or hear a specific deviant stimulus. The whole experiment should take less than an hour to complete.

- During the research you are free to withdraw at any point before the experiment has been completed.

\section{Privacy and Confidentiality}

- We will keep your data and all other information you provide for 5 years at which it will be destroyed.

- Data without identifying names may be used in other, related studies.

- A copy of data without identifying names will remain in the custody of Dr Steven Prime and held in his lab in a secured locker in the School of Psychology.

What happens to the information that you provide?

- The data you provide may be used for one or more of the following purposes:

- The overall findings may be submitted for publication in a scientific journal, or presented at scientific conferences.

- The overall findings may form part of a PhD Thesis, Masters Thesis, or Honours research project that will be submitted for assessment.

If you would like to know the results of this study, they will be available approximately in one to two years and mailed to you. If you wish to know the results please provide us with your email address.

Thank you for considering participation in this research. 


\section{APPENDIX B: Consent Form}

\section{Visual and auditory attentional processing and meditation}

\section{Statement of consent}

I have read the information about this research and any questions I wanted to ask have been answered to my satisfaction.

I agree to participate in this research. I understand that I can withdraw my consent at any time, prior to the end of my participation.

Name:

Signature:

Date:

Subject ID:

Copy to:

[a] participant,

[b] researcher (initial both copies below) 


\section{APPENDIX C: Meditation History Questionnaire}

Please fill in the following information:

Name:

Age:

Gender:

Handedness:

Highest level of education:

The following questions are about your meditation history. Please answer them as accurately as possible.

What type of meditation do you predominantly practice?

How many years have you been practicing meditation?

How many times do you practice meditation in a week?

How long do you spend practicing meditation per week (in minutes)?

How long do you spend meditating per session (in minutes)?

Have you ever been at a meditation retreat?

If so, how many times have you gone to a meditation retreat?

What motivates you to practice meditation?

Please rate how much these sentences apply to your past meditation experience. 
I focus my attention as far as possible to a single point- a mental image, a perceptual object, breath, sound or thought.

$\begin{array}{ll}\text { Strongly Disagree } & \text { Strongly Agree }\end{array}$

$\begin{array}{lllll}1 & 2 & 3 & 4 & 5\end{array}$

I try to concentrate solely on this one item to the exclusion of everything else.

Strongly Disagree

Strongly Agree

$\begin{array}{lllll}1 & 2 & 3 & 4 & 5\end{array}$

I expand my attention/awareness to as many events as possible.

Strongly Disagree

1

2

3

4

Strongly Agree

I consider nothing a distraction.

Strongly Disagree

2

3

4

Strongly Agree

1

5 


\section{APPENDIX D: Debrief Form}

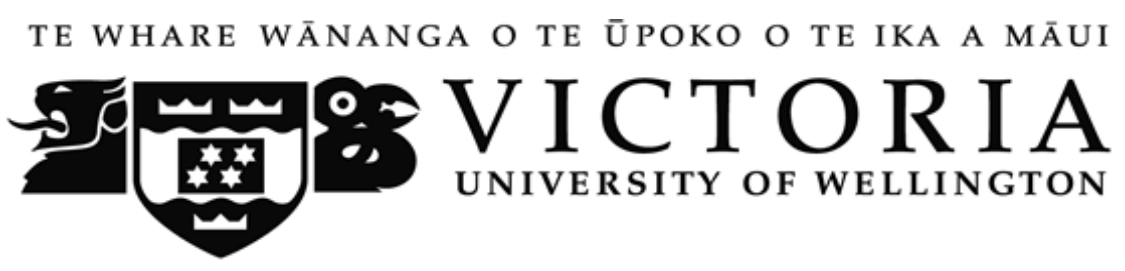

\section{Thank you for participating in our experiment!}

\section{Brief overview of the main aim of our research:}

There is some evidence to suggest that individuals who meditate may have some advantages in visual attentional processing over individuals who do not meditate. The goal of this research is to further investigate these effects by examining individuals who practice different types of meditation.

\section{The experiment you just participated in:}

The aim of this experiment was to determine differences in sustained attention and response inhibition between subjects who practice meditation and those who do not. In this experiment we used a Sustained Attention to Response task. In the original version of the task subjects are required to respond to a rapid stream of digits presented on a screen and then switch their response when a target appears. Due to the repetitive nature of the task, subjects often have short lapses in attention which then lead to errors in detecting the target or responding correctly. We also included an auditory version where subjects responded to tones and a cross-modal version of the task where subjects were required to respond to both tones and digits on the screen. These variations of the original task will help determine whether differences in attentional processing are limited to visual attention or can be extended to auditory attention and the integration of multiple modalities. We want to know if meditators are better at focusing their attention and sustaining it for longer periods than nonmeditators. If this is the case, then we expect meditators would make these errors and be better at identifying the targets when they appear.

Overall this research should better the understanding of the differences in attentional processing for individuals who practice meditation. This knowledge could be used in the development of clinical applications for example the treatment of attentional disorders such as attention deficit disorder or age-related deficits in attention.

If you have any questions about this study or would like to know about other research we are conducting in the lab, please do not hesitate to contact us. We would welcome your participation in any future studies that may be of interest to you, and we look forward to working with you again.

Thank you again for participating in this research!

Ms Paige Badart

Dr John McDowall

Dr Steven Prime
Email: paige.badart@vuw.ac.nz

Email: john.mcdowall@vuw.ac.nz 